\title{
The Neurokinin-1 Receptor is Expressed with Gastrin- Releasing Peptide Receptor in Spinal Interneurons and Modulates Itch
}

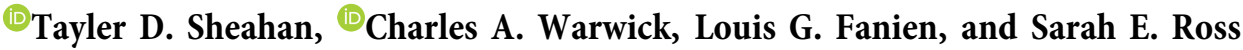 \\ Pittsburgh Center for Pain Research and Department of Neurobiology, University of Pittsburgh, Pittsburgh 15213, Pennsylvania
}

The neurokinin-1 receptor (NK1R; encoded by Tacr1) is expressed in spinal dorsal horn neurons and has been suggested to mediate itch in rodents. However, previous studies relied heavily on neurotoxic ablation of NK1R spinal neurons, which limited further dissection of their function in spinal itch circuitry. To address this limitation, we leveraged a newly developed Tacr1 ${ }^{\text {CreER }}$ mouse line to characterize the role of NK1R spinal neurons in itch. We show that pharmacological activation of spinal NK1R and chemogenetic activation of Tacr1 ${ }^{C r e E R}$ spinal neurons increases itch behavior in male and female mice, whereas pharmacological inhibition of spinal NK1R suppresses itch behavior. We use fluorescence in situ hybridization (FISH) to characterize the endogenous expression of Tacr1 throughout the superficial and deeper dorsal horn (DDH), as well as the lateral spinal nucleus (LSN), of mouse and human spinal cord. Retrograde labeling studies in mice from the parabrachial nucleus (PBN) show that less than $20 \%$ of superficial Tacr ${ }^{\text {CreER }}$ dorsal horn neurons are spinal projection neurons, and thus the majority of Tacr ${ }^{\mathrm{CreER}}$ are local interneurons. We then use a combination of in situ hybridization and ex vivo twophoton $\mathrm{Ca}^{2+}$ imaging of the mouse spinal cord to establish that NK1R and the gastrin-releasing peptide receptor (GRPR) are coexpressed within a subpopulation of excitatory superficial dorsal horn (SDH) neurons. These findings are the first to suggest a role for NK1R interneurons in itch and extend our understanding of the complexities of spinal itch circuitry.

Key words: dorsal horn; GRPR; NK1R; projection neurons; spinal circuitry; spinal cord

\section{Significance Statement}

The spinal cord is a critical hub for processing somatosensory input, yet which spinal neurons process itch input and how itch signals are encoded within the spinal cord is not fully understood. We demonstrate neurokinin-1 receptor (NK1R) spinal neurons mediate itch behavior in mice and that the majority of NK1R spinal neurons are local interneurons. These NK1R neurons comprise a subset of gastrin-releasing peptide receptor (GRPR) interneurons and are thus positioned at the center of spinal itch transmission. We show NK1R mRNA expression in human spinal cord, underscoring the translational relevance of our findings in mice. This work is the first to suggest a role for NK1R interneurons in itch and extends our understanding of the complexities of spinal itch circuitry.

Received July 15, 2020; revised Aug. 25, 2020; accepted Sep. 21, 2020

Author contributions: T.D.S. and S.E.R. designed research; T.D.S., C.A.W., and L.G.F. performed research; T.D.S. analyzed data; T.D.S. wrote the paper.

This work was supported in part by National Institutes of Health Grants T32NS086749 (to T.D.S.), F32NS110155 (to T.D.S.), T32 NS073548 (to C.A.W.), and R01NS096705 (to S.E.R.). We thank all members of the Ross lab for their comments and suggestions, as well as Michael C. Chiang and Justin Chestang for their technical assistance; Dr. Kelly Smith for advice on figure design and Dr. David Baranger for statistical consultation. Select figure panels were created using icons from BioRender. We also thank the donors and their loved ones for making our human studies possible and Dr. Jill Glausier and the Brain Tissue Donation Program at the University of Pittsburgh and the NIH NeuroBioBank for providing us human spinal cord samples.

The authors declare no competing financial interests.

Correspondence should be addressed to Sarah E. Ross at saross@pitt.edu.

https://doi.org/10.1523/JNEUROSCl.1832-20.2020

Copyright $(2020$ the authors

\section{Introduction}

Acute itch, much like pain, serves as a protective warning signal to the body, indicating there is an irritant on the skin that needs to be removed. Itchy stimuli are first detected by primary afferent fibers innervating the skin, which then relay inputs to neurons within the spinal cord dorsal horn, including local interneurons, as well as ascending spinal projection neurons that target brainstem, midbrain, and thalamic structures. However, how these spinal neurons process and encode itch as well as other somatosensory stimuli is not fully understood.

A major unresolved question regarding the spinal circuitry of itch and pain is how to distinguish spinal projection neurons from interneurons. Proposed distinguishing criteria include morphology or genetic markers (Al Ghamdi et al., 2009; Todd, 2010). For instance, the neurokinin-1 receptor (NK1R), an 
excitatory G-protein-coupled receptor that is expressed throughout the nervous system and activated by the neuropeptide substance P (SP), is often highlighted as a selective marker for spinal projection neurons (Todd, 2010; Zhao et al., 2014; Cameron et al., 2015; Häring et al., 2018; Huang et al., 2019; Bardoni et al., 2019). Yet Tacr1, which encodes NK1R, appears to be expressed within multiple excitatory dorsal horn neuron populations recently identified by single cell RNA-sequencing (Häring et al., 2018; Zeisel et al., 2018). Thus, whether NK1R is a true marker of spinal projection neurons requires further investigation.

NK1R has been studied extensively as a putative target for pain relief in preclinical rodent models (Mantyh et al., 1997; Nichols et al., 1999; Vierck et al., 2003; Wiley et al., 2007) but demonstrated negligible clinical success and was quickly dropped as a therapeutic target (Hill, 2000; Boyce and Hill, 2004). Intriguingly, NK1R expressed on spinal neurons has recently emerged as a potential therapeutic target for chronic itch. Neurotoxic ablation studies show that loss of NK1R spinal neurons reduces scratching in rodents (Carstens et al., 2010; Akiyama et al., 2015; Acton et al., 2019). However, the effect of modulation of NK1R spinal neurons in an intact spinal circuit remains unclear. Furthermore, little is known about which NK1R neurons are involved in itch processing or where they fit within the current model of itch transmission.

The current model of itch spinal circuitry positions neurons that express the gastrin-releasing peptide receptor (GRPR) as a gate for itch input (Sun and Chen, 2007; Sun et al., 2009; Liu et al., 2019; Pagani et al., 2019; Lay and Dong, 2020). We explored the possibility that NK1R and GRPR neurons might represent an overlapping dorsal horn neuron subpopulation that modulates itch.

In this study, we demonstrate that activation of NK1R spinal neurons potentiates itch, while inhibition of NK1R attenuates itch. We highlight that, despite being widely used as a selective marker of spinal projection neurons, NK1R is expressed predominately in interneurons within the dorsal horn. Moreover, we reveal a likely role for NK1R interneurons in spinal itch transmission, and that these NK1R neurons comprise a subpopulation of excitatory GRPR interneurons within the superficial dorsal horn $(\mathrm{SDH})$.

\section{Materials and Methods \\ Donors}

Human spinal cord tissue was obtained from the University of Pittsburgh NIH NeuroBioBank Brain and Tissue Repository in compliance with the University of Pittsburgh's Committee for the Oversight of Research involving the Dead and Institutional Review Board for Biomedical Research. Spinal cord tissue from the C1 spinal segment was collected from adult human organ donors (donor 1: white 45-year-old male, donor 2: white 44-year-old female), flash frozen, and stored at $-80^{\circ} \mathrm{C}$ until use.

\section{Animals}

All animals were cared for in compliance with the National Institutes of Health guidelines and approved by the University of Pittsburgh Institutional Animal Care and Use Committee. Experiments were conducted on both male and female mice, with littermates randomly assigned to experimental groups. Adult, C57BL/6 mice were obtained from Charles River (strain 027) and allowed to acclimate to the University of Pittsburgh's vivarium for at least one week before beginning experimentation. Previously generated Tacrl ${ }^{\mathrm{CreER}}$ mice (also referred to as NK1R-CreER mice; Huang et al., 2016) were bred in house and maintained on a C57BL/6 background. For histology and in situ hybridization studies, Tacr $1^{\text {CreER }}$ mice were crossed with Cre-dependent $\operatorname{Ros}^{t d T}$ reporter mice (Jackson Laboratory, stock no. 007909). For two- photon $\mathrm{Ca}^{2+}$ imaging studies, Vglut2 ${ }^{\mathrm{Cre}}$ mice (The Jackson Laboratory, stock no. 016963) were crossed with Cre-dependent Rosa ${ }^{G C a M P 6 s}$ mice (The Jackson Laboratory, strain no. 028866).

Mice were housed in cages of four (males) or five (females) in the animal facility under a $12 / 12 \mathrm{~h}$ light/dark cycle (7 A.M. to 7 P.M.) and provided food and water ad libitum. Cages were lined with woodchip bedding. Behavioral studies on and tissue harvesting from C57BL/6 and Tacr $1^{\text {CreER }}$ mice began when animals were six to eight weeks old. Spinal cord $\mathrm{Ca}^{2+}$ imaging studies were similarly performed on six- to eightweek-old Vglut2 ${ }^{\text {Cre }}::$ Rosa $^{\text {GCaMP6s }}$ mice.

\section{Tamoxifen administration}

Tamoxifen (Sigma, T5648) was dissolved in corn oil (Sigma, C8267) by shaking it at $37^{\circ} \mathrm{C}$ to create a $20-\mathrm{mg} / \mathrm{ml}$ stock solution. This solution was stored, protected from light, at $4^{\circ} \mathrm{C}$ for up to one week. Tamoxifen was administered intraperitoneally at $75 \mathrm{mg} / \mathrm{kg}$ for five consecutive days. Tacr ${ }^{\text {CreER }}$;Rosa ${ }^{t d T}$ mice were injected with tamoxifen at $\sim 2.5$ weeks of age. Intraspinal viral injections were performed on approximately fourweek-old Tacr $1^{\text {CreER }}$ mice, and tamoxifen administration began $48 \mathrm{~h}$ after surgery.

\section{Intraspinal viral injections}

Mice were anesthetized with a ketamine/xylazine cocktail $(87.5 \mathrm{mg} / \mathrm{kg} /$ $12.5 \mathrm{mg} / \mathrm{kg}$, i.p.); Ophthalmic ointment (Dechra, 12920060) was applied to the eyes. The back was shaved, and the local antiseptic betadine (Fischer Scientific, 19-061617) was applied to the skin. Using a scalpel, a skin incision was made over the T12-L3 vertebrae. Small scissors were used to cut through fascia, and \#5 forceps were used to separate muscle from the T12-T13 vertebrae, exposing the intervertebral space above the left L3/L4 spinal segments (Blizzard et al., 2016; Haenraets et al., 2018). Laminectomies were avoided to minimize spinal cord trauma. A glass capillary was carefully lowered down $300 \mu \mathrm{m}$ from the surface of the dura, taking care to leave the posterior spinal artery intact. Once lowered, $500 \mathrm{nl}$ of virus was injected at a flow rate of $5 \mathrm{nl} / \mathrm{s}$ using a NanoInject III Programmable Nanoliter Injector (Drummond Scientific Company) mounted on a stereotax (Kopf, Model 942), and the glass capillary was left in place for a total of $5 \mathrm{~min}$ and then slowly withdrawn. The skin incision was closed with 5-0 vicryl suture (Ethicon, VCP493G). After surgery, mice were injected subcutaneously with $0.3 \mathrm{mg} / \mathrm{kg}$ buprenorphine and $5 \mathrm{mg} / \mathrm{kg}$ ketoprofen and allowed to recover on a heating pad. On average, unilateral intraspinal viral injections resulted in 1.6 $\mathrm{mm}$ of rostral-caudal viral spread, spanning the L3-L5 spinal segments. Behavioral tests began four weeks after viral injection to allow for maxi$\mathrm{mal}$ and stable viral expression. At the conclusion of behavioral studies, intraspinal viral injections were confirmed with histology (described in Immunohistochemistry). One Tacr1::hM3Dq-mCherry mouse was excluded from analyses because there was no hM3Dq-mCherry detected within the lumbar spinal cord.

\section{Viral constructs}

For chemogenic and anterograde tracing studies, an adeno-associated virus (AAV2) coding for Cre-dependent hM3Dq-mCherry, AAV2-hSynDIO-hM3D $(\mathrm{Gq})$-mCherry $(5.8 \times 1012 \mathrm{particles} / \mathrm{ml}$; Addgene, catalog \#44361-AAV2, lot \#V12832) was injected intraspinally; these animals are referred to as Tacr1::hM3Dq-mCherry mice throughout the text. AAV Cre-dependent fluorescent reporters: AAV2-EF1a-DIO-eYFP $(4.6 \times 1012$ particles/ml; UNC Vector Core, lot \#AV4842E; 7 mice) or AAV2-hSynDIO-mCherry $(4.7 \times 1012$ particles/ml; Addgene, catalog \#50459-AAV2, lot \#V27924; three mice) were used as controls. For simplicity, mice that received either control virus are referred to as Tacr1::control throughout the text. Viruses were stored in aliquots at $-80^{\circ} \mathrm{C}$ until use.

\section{Chemogenetic studies}

Based off of previous publications (Peirs et al., 2015; Jendryka et al., 2019), mice were administered the water-soluble version of clozapine $\mathrm{N}$ oxide (CNO), CNO hydrochloride (Tocris, catalog \#6329), which was dissolved in sterile saline and delivered at $5 \mathrm{mg} / \mathrm{kg}$, intraperitoneally. Behavioral testing was completed within $2.5 \mathrm{~h}$ of $\mathrm{CNO}$ injection. Baseline nociceptive withdrawal testing (heat, cold, mechanical) took 
place at least $1 \mathrm{~d}$ before $\mathrm{CNO}$ administration. Following $\mathrm{CNO}$ administration, some behavioral assays were paired (spontaneous behavior and heat sensitivity, cold sensitivity and chloroquine-induced itch) to minimize the number of $\mathrm{CNO}$ injections experienced by an individual mouse. All nociceptive withdrawal assays were performed at least $25 \mathrm{~min}$ following $\mathrm{CNO}$ administration to allow sufficient time for $\mathrm{CNO}$ to take effect and for the mice to stop exploring the testing apparatus.

\section{Behavioral studies}

All behavioral studies were performed by the same tester to minimize experimenter variability. The experimenter was blind to all experimental conditions throughout data acquisition and analysis. Each behavioral study was performed on two different cohorts of mice for a total of 8-10 mice per experimental group across cohorts. Injection sites were shaved at least $24 \mathrm{~h}$ before testing. On testing days, mice were acclimated to the testing apparatus in Plexiglas boxes for at least $30 \mathrm{~min}$ before the start of testing. When applicable, once all animals in a cohort were injected, the experimenter left the room. Experiments were performed during the light cycle between 10 A.M. and 5 P.M.

\section{Intrathecal injections}

Intrathecal injections were performed on awake, behaving animals to avoid effects of anesthesia on behavior as previously described, with minor modifications (Kardon et al., 2014; Njoo et al., 2014). Mice were pinned down by the pelvic girdle, and drugs were delivered between the L5 and L6 vertebrae using a 30-G needle attached to a $25-\mu$ l Hamilton syringe. Successful insertion of the needle into the intervertebral space was indicated by a sudden lateral movement of the tail. Thereafter, the drug was slowly injected at a rate of $1 \mu \mathrm{l} / \mathrm{s}$, and the needle was held in place for an additional $5 \mathrm{~s}$ to minimize backflow. Only animals with successful injections were included in data analysis. The following drugs were delivered in a volume of $5-\mu l$ sterile saline: SP (400 ng, Sigma, S6883), selective NK1R agonist GR 73, 632 (40 ng, Tocris, 1669), GRP (286 ng, Tocris, 1789), and NK1R antagonist CP 99994 (18 $\mu \mathrm{g}$, Tocris, 3417). Sterile saline was delivered as a vehicle control.

SP-evoked, selective NK1R agonist-evoked, and GRP-evoked behavior Mice were injected intrathecally with SP, the selective NK1R agonist GR 73,632 , or vehicle as described above. Immediately following injection, animals were returned to their behavior boxes and their activity was video recorded to assess spontaneous behaviors. The three most prevalent behaviors elicited by SP and GR 73, 632 included scratch bouts, biting bouts, and head grooming. A scratch bout was defined as a rapid back and forth movement of the hindlimb (in this case, usually directed toward the abdomen) that ended in either licking/biting of the hindpaw or returning the hindpaw to the floor. A biting bout was defined as contact of the snout with the abdomen, ending with the mouse raising its head away from its body. Head grooming was defined as a single bilateral forepaw stroke, moving from the caudal to rostral end of the cheek, a stereotyped movement within mouse self-grooming chains (Kalueff et al., 2016). The number of scratch bouts, biting bouts, and head grooming events were quantified for $5 \mathrm{~min}$ (SP) or $20 \mathrm{~min}$ (GR 73, 632) following intrathecal injection. In a separate experiment, mice were injected intrathecally with GRP and scratch bouts, biting bouts, and head grooming events were quantified for the subsequent $30 \mathrm{~min}$.

\section{Chloroquine-induced itch behavior}

In pharmacological inhibition studies, chloroquine $(100 \mu \mathrm{g}$ in $5-\mu$ l sterile saline, Sigma, C6628) was administered intradermally into the calf 15 min following intrathecal injection of either CP 99994 or saline. Chemogenetic studies similarly used the calf model of itch (LaMotte et al., 2011; Akiyama et al., 2014) because intraspinal viral injections were localized to the lumbar spinal cord. Chloroquine ( $100 \mu \mathrm{g}$ in $5-\mu \mathrm{l}$ sterile saline) was administered intradermally into the calf ipsilateral to intraspinal viral injection $120 \mathrm{~min}$ after the injection of CNO. For both studies, mice were video recorded for $30 \mathrm{~min}$ following the chloroquine injection, and the duration of time spent biting the calf was quantified off-line in 5-min intervals.

\section{CNO-evoked spontaneous behavior}

Immediately after administration of $\mathrm{CNO}$, mice were video recorded for $60 \mathrm{~min}$. The duration of all behaviors directed toward the hindlimb ipsilateral to the intraspinal viral injection, including hindpaw licking, lifting, flinching, as well as calf or haunch biting or grooming, was quantified off-line in 5-min intervals.

\section{Heat sensitivity (Hargreaves test)}

Animals were acclimated on a glass plate held at $30^{\circ} \mathrm{C}$ (IITC). Beginning 60 min following $\mathrm{CNO}$ administration, a radiant heat source (active intensity $=10 \%$; intermittent intensity $=3 \%$ ) was applied to the glass beneath the hindpaw and latency to paw withdrawal was recorded (Hargreaves et al., 1988). Three trials were conducted on each paw, with at least $5 \mathrm{~min}$ between testing the opposite paw and at least $10 \mathrm{~min}$ between testing the same paw. A cut off latency of $20 \mathrm{~s}$ was set to avoid tissue damage. Values across trials were averaged to determine withdrawal latency of each paw.

\section{Cold sensitivity (cold plantar assay)}

Cold sensitivity was measured as previously described (Brenner et al., 2012 , 2015). Mice were acclimated to a $1 / 4$ " glass plate and a cold probe was made by packing finely crushed dry ice into a modified $3 \mathrm{ml}$ syringe $1 \mathrm{~cm}$ in diameter. Beginning $25 \mathrm{~min}$ after $\mathrm{CNO}$ administration, the cold probe was applied to the glass beneath the plantar surface of the hindpaw and the latency to paw withdrawal was recorded. Three trials were conducted on each hindpaw, with $5 \mathrm{~min}$ between trials on opposite paws, and $10 \mathrm{~min}$ between trials on the same paw. A cut off latency of $20 \mathrm{~s}$ was used to prevent tissue damage. Withdrawal latencies for each paw were determined by averaging values across trials.

\section{Mechanical sensitivity (von Frey)}

Mechanical sensitivity was measured using the simplified up-down (SUDO) method of the von Frey test (Bonin et al., 2014). Beginning 40 min following $\mathrm{CNO}$ administration, calibrated von Frey filaments (North Coast Medical Inc.) were applied to the plantar surface of the hindpaw for $2 \mathrm{~s}$. For each trial, a total of five filament applications was performed and used to calculate the paw withdrawal threshold. Three trials were conducted on each hindpaw, with $5 \mathrm{~min}$ between trials on opposite paws, and $10 \mathrm{~min}$ between trials on the same paw. The average paw withdrawal threshold from all three trials are reported in units of pressure $\left(\mathrm{g} / \mathrm{mm}^{2}\right)$ to account for differences in filament surface areas.

\section{Capsaicin-induced nocifensive behavior}

Forty minutes after CNO injection, $10 \mu \mathrm{l}$ of $0.001 \%$ capsaicin (dissolved in $10 \%$ ethanol, $0.5 \%$ Tween 80 in sterile saline; Sigma, M2028) was injected intraplantar into the hindpaw ipsilateral to the intraspinal viral injection and mice were video recorded for $15 \mathrm{~min}$. The duration of nocifensive behaviors including lifting, licking, or shaking the hindpaw was quantified off-line in 5-min intervals.

\section{Stereotaxic injection and retrograde labeling}

Mice were anesthetized with isoflurane (induction: $4 \%$, maintenance: $2 \%$ ) and head-fixed in a stereotaxic frame (Kopf, model 942). Ophthalmic ointment was applied to the eyes, the scalp was shaved, and local antiseptics (betadine and ethanol) were applied before using a scalpel to make a midline incision to expose the skull. The skull was leveled using cranial fissures as landmarks. To target the lateral parabrachial nucleus (lPBN), a drill bit (Stoelting, 514551) was used to create a burr hole at the following empirically derived coordinates: AP $-5.11 \mathrm{~mm}$, ML $\pm 1.25 \mathrm{~mm}$, DV $-3.25 \mathrm{~mm}$. A glass capillary was carefully lowered through the burr hole to the injection site, and a Nanoinject III was used to deliver $500 \mathrm{nl}$ of the fluorescently conjugated retrograde tracer cholera toxin subunit B-Alexa Fluor 647 (CTB, Thermofisher C34778, $1 \mathrm{mg} / \mathrm{ml}$ ) at a rate of $5 \mathrm{nl} / \mathrm{s}$. The glass capillary was left in place for a total of $5 \mathrm{~min}$ and then slowly withdrawn. The scalp was sutured closed with 5-0 vicryl suture. After surgery, mice were injected subcutaneously with $0.3 \mathrm{mg} / \mathrm{kg}$ buprenorphine and $5 \mathrm{mg} / \mathrm{kg}$ ketoprofen and allowed to recover on a heating pad. Immunohistochemistry studies were conducted at least $10 \mathrm{~d}$ after CTB injection into the IPBN to allow for maximal retrograde labeling of spinal projection neurons. 
Immunohistochemistry

For histology studies, Tacr ${ }^{\text {CreER }}$ mice expressing hM3Dq-mCherry (virally-mediated) and CTB-647 or tdTomato (tdT; constitutive reporter) were anesthetized with urethane and perfused with $4 \%$ paraformaldehyde. Lumbar spinal cord, dorsal root ganglia, and brain were dissected and postfixed in $4 \%$ paraformaldehyde at $4^{\circ} \mathrm{C}$ for either $2 \mathrm{~h}$ (spinal cord, dorsal root ganglia) or overnight (brain). Tissues were washed in PBS and cryoprotected in $30 \%$ sucrose before cryosectioning. When directly mounted onto slides, $40-\mu \mathrm{m}$ transverse spinal cord and $20-\mu \mathrm{m}$ dorsal root ganglia sections were collected. When stained as free-floating sections, $60-\mu \mathrm{m}$ transverse spinal cord and $60-\mu \mathrm{m}$ coronal brain sections were collected and stored in PBS with $0.01 \%$ sodium azide. Tissues were incubated in a blocking solution consisting of $10 \%$ normal donkey serum (Jackson ImmunoResearch, 017-000-121) and 0.3\% Triton X-100 solution (Sigma, 93443) in PBS for $1 \mathrm{~h}$ at room temperature. The primary antibodies rabbit anti-RFP (Rockland, 600-401-379), guinea pig anti-cFOS (Synaptic Systems, 226005), and rabbit anti-NK1R (Sigma, S8305, 1:10,000) were diluted in antibody buffer consisting of 5\% normal donkey serum and $0.3 \%$ Triton X-100 in PBS. Tissue was incubated in primary antibody overnight at $4^{\circ} \mathrm{C}$, then washed in PBS three times for $10 \mathrm{~min}$. The secondary antibodies donkey anti-rabbit Alexa Fluor 555 (Invitrogen, A-31572, 1:500) and goat anti-guinea pig Alexa Fluor 488 (Invitrogen, SA5-10094, 1:500), were diluted in antibody buffer and applied for $1 \mathrm{~h}$ (mounted sections) or $2 \mathrm{~h}$ (free-floating sections). Finally, tissues were washed again in PBS three times for $10 \mathrm{~min}$ and mounted with Prolong Gold with DAPI (Invitrogen, P36931).

In experiments evaluating expression of AAV2-hSyn-DIO-hM3D (Gq)-mCherry in the spinal cord and dorsal root ganglia, sections were mounted and stained directly on Super Frost Plus slides (Fisher Scientific, 12-550-15). In studies visualizing NK1R immunoreactivity, as well as the brain targets of Tacr ${ }^{\text {CreER }}$ spinal projection neurons, sections were stained free-floating. In experiments evaluating the percentage of Tacr1 ${ }^{\text {CreER }}$ spinoparabrachial neurons versus interneurons, only mice with confirmed on-target CTB injections into the PBN were analyzed. CTB-647 and tdT signals were not amplified.

\section{cFOS induction}

To verify that systemic CNO administration caused hM3Dq activation (and in turn neuronal activation), Tacr1::hM3Dq-mCherry and Tacr1:: control mice were injected with $5 \mathrm{mg} / \mathrm{kg}$ CNO intraperitoneally $9 \mathrm{~min}$ before perfusion with $4 \%$ paraformaldehyde, in line with the time course of behavioral studies. Spinal cord tissue was processed and immunostained for as described above.

\section{Fluorescence in situ hybridization (FISH; RNAscope)}

Mice were anesthetized with isoflurane and rapidly decapitated. The L3/ L4 spinal cord segments were quickly removed within $5 \mathrm{~min}$, placed into OCT, and flash frozen using 2-methylbutane chilled on dry ice. Tissue was kept on dry ice until cryosectioning. Mouse lumbar and human cervical spinal cord samples were cryosectioned at $15 \mu \mathrm{m}$, mounted directly onto Super Frost Plus slides, and FISH studies were performed according to the protocol for fresh frozen samples using the RNAscope Multiplex Fluorescent v2 Assay (Advanced Cell Diagnostics, 323100) with minor modifications. Briefly, spinal cord tissue was dehydrated with ethanol and fixed for $15 \mathrm{~min}$ in ice-cold $4 \%$ PFA. Sections were treated with hydrogen peroxide for $10 \mathrm{~min}$ at room temperature, followed by Protease IV for $15 \mathrm{~min}$ at room temperature. Probes for Tacr1 (Advanced Cell Diagnostics, Mm-Tacr1, 428781), tdTomato (-tdTomato, 317041), Grpr (Mm-Grpr, 517631), Slc17a6 (referred to here as Vglut2, Mm-Slc16a6, 319171), and TACR1 (Hs-TACR1, 310701) were hybridized for $2 \mathrm{~h}$ at $40^{\circ} \mathrm{C}$ in a humidified oven, then stored overnight in $5 \times$ saline sodium citrate. After rinsing in wash buffer, a series of incubations was then performed to amplify and develop hybridized probe signal using TSA Plus Fluorophores (PerkinElmer, NEL741001KT and NEL744E001KT). Slides were mounted with Prolong Gold with DAPI.

\section{Imaging and quantification}

Sections were imaged at full tissue-thickness using an upright epifluorescent microscope (Olympus BX53 with UPlanSApo $4 \times, 10 \times$, or $20 \times$ objectives), a confocal microscope (Nikon A1R with $20 \times$ or $60 \times$ oilimmersion objectives), or a slide scanner (Olympus VS200, 40× oil objective). All analysis was completed off-line using FIJI software (ImageJ, NIH). For FISH experiment quantification, two to four spinal cord hemisections/mouse were manually quantified from four to five mice per experiment, and three spinal cord hemisections were quantified from two human donors. A positive cell was defined as a cell with a clearly defined nucleus and fluorescent signal forming a ring around the nucleus. To quantify the number of recombined Tacr1::hM3Dq-mcherry neurons from the onset to offset of viral spread, neurons from every sixth section (thus, every $240 \mu \mathrm{m}$ ) were quantified and their laminar distribution was noted ( $n=7$ mice). For CTB-backlabeled and cFOS neuron quantification, four to five sections/mouse were manually counted from three to four mice per condition.

Analyses of the spatial distribution of Tacrl, Tacr1::hM3DqmCherry, and CTB neurons in mouse and human used the following boundaries: In mouse, the SDH was defined as the region $65 \mu \mathrm{m}$ below the surface of the dorsal horn gray matter, roughly corresponding to laminae $\mathrm{I}$ and $\mathrm{II}_{\mathrm{o}}$. This distance was determined by measuring the distance from the surface of the dorsal horn gray matter to the bottom of the Grpr band in FISH sections, based on previous studies demonstrating that GRPR neurons are restricted to the SDH (Sun and Chen, 2007; Sun et al., 2009; Bardoni et al., 2019). In the human spinal cord, the SDH was defined as the region bounded by the surface of the dorsal horn gray matter and the bottom of the substantia gelatinosa, roughly corresponding to lamina I-II. In both mouse and human, the deeper dorsal horn (DDH) consisted of the remaining lamina, roughly III-VI of the dorsal horn. Lateral spinal nucleus (LSN) neurons were defined as those located lateral to the dorsal horn gray matter, within the dorsolateral funiculus.

\section{Two-photon $\mathrm{Ca}^{2+}$ imaging}

As described previously (Hachisuka et al., 2016), the C2-S6 spinal cord segments were dissected from $\mathrm{Vglut}_{2}{ }^{\mathrm{Cre}} ;$ Rosa $^{G C a M P 6 s}$ mice and placed in a Sylgard recording chamber designed for pharmacology providing fast and uniform fluid exchange. Using a Leica SP-5 multiphoton microscope coupled to a Chameleon Ultra tunable Ti:Sapphire laser and a Leica $20 \times$ (NA 1.00), the lumbar (L1-3) superficial gray matter (0$100 \mu \mathrm{m}$, encompassing laminae I and II) was imaged at three to four $Z$ planes at $1 \mathrm{~Hz}$, sampling up to $\sim 200$ excitatory neurons in a given experiment within a typical $\sim 0.13 \mathrm{~mm}^{2}$ field of view. Throughout imaging experiments, the recording chamber was continuously perfused with normal artificial CSF (aCSF) solution ( $117 \mathrm{~mm} \mathrm{NaCl}, 3.6 \mathrm{~mm} \mathrm{KCl}, 2.5$ mм CaCl $2,1.2 \mathrm{~mm} \mathrm{MgCl}_{2}, 1.2 \mathrm{~mm} \mathrm{NaH}_{2} \mathrm{PO}_{4}, 25 \mathrm{~mm} \mathrm{NaHCO}$, and 11 mu glucose) saturated with $95 \% \mathrm{O}_{2}$ and $5 \% \mathrm{CO}_{2}$ at $30^{\circ} \mathrm{C}$. TTX (500 nм, Tocris 1069) was added to the bath to identify neurons that responded directly to SP (1 $\mu \mathrm{M}$, Sigma, S6883) or GRP (300 nм, Tocris, 1789), which were applied $5 \mathrm{~min}$ apart, in random order. Agonist concentrations were selected based on previous in vitro spinal cord physiology studies (Marvizón et al., 1997; Nakatsuka et al., 2005; Aresh et al., 2017; Liu et al., 2019; Pagani et al., 2019). At the conclusion of experiments, modified aCSF containing $30 \mathrm{~mm} \mathrm{~K}^{+}$was perfused onto the spinal cord to activate and visualize all GCaMP6-expressing neurons, as well as to assess their viability.

For image processing, a Suite $2 \mathrm{p}$ pipeline, custom tuned for dorsal horn neurons, was used for image registration and signal extraction. ImageJ was used for all other image processing. Responders were defined as neurons that had both a peak value in $\Delta \mathrm{F} / \mathrm{F}$ of $\geq 80 \%$ and a $\Delta \mathrm{F} / \mathrm{F}$ of $\geq 4$ SDs calculated from the $30 \mathrm{~s}$ before drug perfusion. Within GRPresponsive cells, $\mathrm{Ca}^{2+}$ oscillations, likely arising from intracellular stores (Karlsson and Ahrén, 1999), were noted, and if an individual neuron's oscillations extended into the period of time during SP application, the cell was excluded from analysis.

\section{Statistical analyses}

Microscope Excel, GraphPad Prism, and the R treemap package were used for data organization and statistical analyses. Statistical significance was indicated by $p \leq 0.05$ determined using Student's $t$ test, MannWhitney $U$ test, two-way repeated measures (RM) ANOVA, two-way ANOVA, or a mixed-effects model. The Holm-Sidak test was used to 
correct for multiple comparisons, when applicable. See figure legends for experiment-specific details. All values are presented as mean \pm SEM.

\section{Results}

NK1R spinal neurons modulate itchrelated behaviors

When injected into the skin, SP can elicit itch-related behaviors such as scratching (Azimi et al., 2016, 2017). We tested whether SP similarly acts as a pruritogen within the spinal cord. C57BL/6 mice were injected intrathecally with SP or saline, and their spontaneous behaviors were quantified (Fig. 1A). Compared with control mice injected with saline, mice injected with SP exhibited significantly more spontaneous itch-related behaviors, including scratch bouts, biting bouts, and head grooming events (Fig. $1 B)$. The effect of SP was immediate and subsided within $5 \mathrm{~min}$, likely because of rapid internalization of NK1R on binding of SP (Mantyh et al., 1995; Wang and Marvizón, 2002).

Because SP can bind at lower affinities to other tachykinin receptors that are expressed on spinal neurons (Ständer and Luger, 2015)-NK2R and NK3R-we tested whether selective activation of NK1R similarly elicited spontaneous itchrelated behaviors. Indeed, like SP, intrathecal injection of the selective NK1R agonist GR 73, 632 caused a significant increase in the number of scratch bouts, biting bouts, and head grooming responses relative to saline (Fig. $1 C$ ). In contrast to SP, the effects of GR 73, 632 were longer lasting, and continued for at least 20 min, suggesting that NK1R internalization does not occur as rapidly on binding of GR 73, 632.

These findings are in agreement with previous reports that intrathecal administration of SP elicits scratching and biting behavior in rodents (Hylden and Wilcox, 1981; Seybold et al., 1982; Ruzza et al., 2014). In some cases, SP-induced scratching and biting have been referred to as pain-related behaviors. However, the behavioral responses to intrathecal injection of SP and GR 73, 632 closely resembled those to intrathecal injection of the well-established pruritogen, GRP. Comparing mice that were injected intrathecally with either GRP or NK1R agonists, we determined the percentage of total behavioral responses represented by scratch bouts, biting bouts, and head grooming for each agonist. Notably, there was no difference in the percentage of scratch bouts, biting bouts, and head grooming events elicited by SP, GR, 73, 632, or GRP (Fig. 1D). Thus, we favor the idea that like GRP, behaviors triggered by spinal NK1R activation are itchrelated. Increased head grooming is an interesting observation in these experiments because it is not a canonical itch-related behavior. It might, however, represent an indirect response to
B

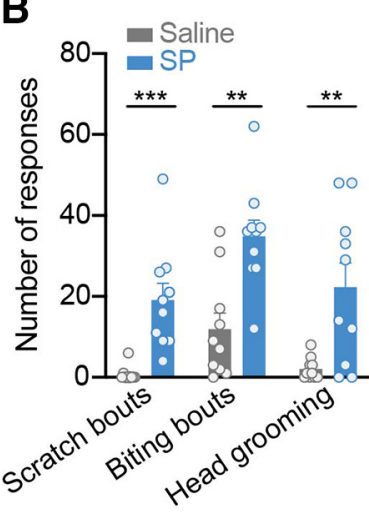

C

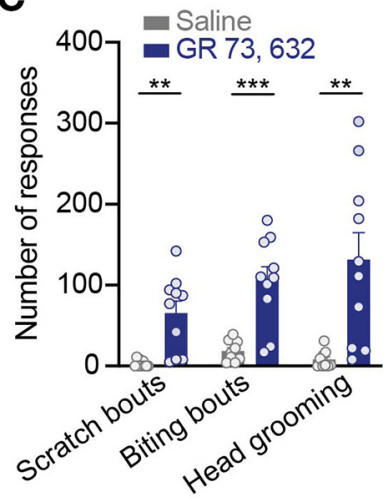

$\mathbf{F}$
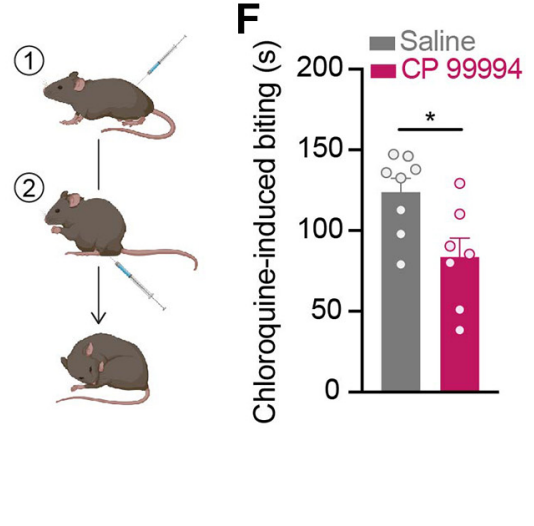

Figure 1. Spinal NK1R modulates itch. $\boldsymbol{A}, \mathrm{C} 57 \mathrm{BL} / 6$ mice were injected intrathecally with either saline, SP $(400 \mathrm{ng})$, the selective NK1R agonist GR 73, 632 (40 ng), or GRP (285 ng), and three spontaneous itch-related behaviors were quantified: bouts, and head grooming responses than saline in the 5 min immediately following injection (Student's $t$ test, Holm-Sidak

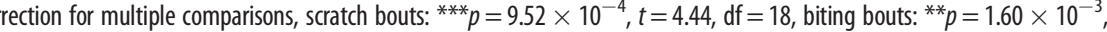
increased the number of scratch bouts, biting bouts, and head grooming responses compared with saline in the ${ }^{* *} p=2.01 \times 10^{-3}, t=3.96, \mathrm{df}=17$, biting bouts: ${ }^{* * *} p=5.73 \times 10^{-4}, t=4.74, \mathrm{df}=17$, head grooming: ${ }^{* *} p=2.66 \times$ $10^{-3}, t=3.51, \mathrm{df}=17 ; n=9-10$ mice/group). $\boldsymbol{D}$, The percentage of scratch bouts, biting bouts, and head grooming established pruritogen, GRP (two-way ANOVA, behavioral response $\times$ agonist, $p=0.296, F_{(4,78)}=1.25, n=9-10$ mice/ group). Behavioral responses to GRP were quantified for the $30 \mathrm{~min}$ immediately following injection. $\boldsymbol{E}, \mathrm{C} 57 \mathrm{BL} / 6$ mice were dermal injection of chloroquine into the calf $(100 \mu \mathrm{g} / 5 \mu \mathrm{l})$, and the duration of site-directed biting was quantified. $\boldsymbol{F}$, athecal pretreatment with CP 99994 significantly decreased the duration of chloroquine-induced biting compared with saline (Student's $t$ test, ${ }^{*} p=0.016, t=2.77, \mathrm{df}=13 ; n=7-8$ mice). SP, substance P; GRP, gastrin-releasing peptide. Data are shown as mean \pm SEM, with open circles representing individual mice.

itch. There is growing evidence that itch is aversive and increases anxiety in rodent models (Samineni et al., 2019; Sanders et al., 2019), and increased anxiety is in turn associated with heightened rodent self-grooming (Kalueff and Tuohimaa, 2004; Kalueff et al., 2016).

Since activation of spinal NK1R potentiated itch, we then asked whether inhibition of spinal NK1R blocked itch. We intrathecally delivered the NK1R antagonist CP 99994 at a dose previously shown to inhibit pain behaviors (Bonin and De Koninck, 2014), then injected the pruritogen chloroquine intradermally into the calf to elicit itch. When pruritogens are injected into the calf, mice bite the injection site (LaMotte et al., 2011). As expected, compared with mice pretreated with saline, mice pretreated with CP 99994 exhibited significantly less calf-direct biting in response to chloroquine (Fig. 1E), complementing previous findings (Carstens et al., 2010; Akiyama et al., 2013, 
2015; Acton et al., 2019). Taken together, these results indicate NK1R spinal neurons mediate itch behaviors.

Tacr1 is broadly expressed within the spinal cord dorsal horn After establishing that NK1R spinal neurons drive itch-related behaviors, our overarching goal was to understand which NK1R neurons within the spinal cord contribute to itch. As a first step, we sought to characterize the laminar distribution of NK1R neurons within the mouse spinal cord dorsal horn. We performed FISH for Tacr1, the gene encoding NK1R, and quantified the number of Tacrl-expressing neurons located within the SDH, DDH, and LSN (Fig. 2A,B).

Tacr1 neurons were spatially distributed throughout the dorsal horn. However, only a fraction of Tacr1 neurons (24.2 \pm $4.3 \%$ ) were localized to the $\mathrm{SDH}$, with the majority of Tacr1 neurons $(71.8 \pm 4.8 \%)$ instead found in the DDH. A small percentage of Tacr1 neurons were located in the LSN (4.0 $\pm 1.5 \%)$. Importantly, FISH was also performed on spinal cord tissue from a homozygous Tacrl ${ }^{\text {CreER }}$ mouse, which is homozygous null (NK1R-/-), and we detected negligible signal, confirming the specify of the Tacrl probe (Fig. 2C).

Our findings demonstrate that Tacrl is broadly expressed throughout the SDH and DDH. However, NK1R has long been used as a marker for ascending spinal projection neurons that reside within laminae I and III-V of the dorsal horn, as well as the LSN (Todd et al., 2000; Spike et al., 2003; Polgár et al., 2010; Cameron et al., 2015). Spinal projection neurons of the anterolateral tracts are an extremely sparse population, comprising no more than $5 \%$ and $0.1 \%$ of total neurons within the $\mathrm{SDH}$ and DDH, respectively (Todd, 2010), but the total numbers of Tacr1 neurons in our study suggest more widespread expression. Thus, these results suggest that Tacrl expression is not limited to spinal projection neurons, consistent with previous reports (Todd et al., 1998; Al Ghamdi et al., 2009).

\section{TACR1 is primarily expressed in the SDH of the human spinal cord}

We next asked whether NK1R spinal neurons may similarly be involved in the spinal processing of itch input in humans. Analogous to mouse studies, we performed FISH for TACR1 on human spinal cord tissue and quantified the number of TACR1expressing neurons located within the SDH, DDH, and LSN (Fig. 2D-F).

Interestingly, in contrast to mouse, the majority of TACR1 neurons $(88.8 \pm 1.0 \%)$ were localized to the $\mathrm{SDH}$, although a small percentage were detected within the DDH $(10.5 \pm 1.1 \%)$ and LSN $(0.7 \pm 0.2 \%)$. Nevertheless, these findings establish that TACR1 expression within the $\mathrm{SDH}$ is evolutionarily conserved between mouse and human and suggest that NK1R may well positioned in the spinal cord to modulate itch in humans.

\section{Tacr $1^{\text {CreER }}$ captures a subpopulation of Tacr1 spinal dorsal horn neurons}

Dissection of the role of NK1R neurons in spinal processing of itch has been limited by a lack of genetic tools that selectively target NK1R neurons. To gain genetic access to Tacr1 neurons, we previously generated a Tacr ${ }^{\text {CreER }}$ knock-in mouse line (Huang et al., 2016). However, the efficiency and specificity of Tacr ${ }^{\text {CreER }}$ mediated recombination within spinal neurons was unknown.

To assess the efficiency and specificity of our Cre line, we crossed Tacr1 ${ }^{\text {CreER }}$ mice to mice harboring a Cre-dependent tdT fluorescent reporter expressed under control of the Rosa locus $\left(\right.$ Tacr ${ }^{\text {CreER }}$;Rosa ${ }^{t d T}$; Fig. 2G). Immunohistochemistry and FISH revealed tdT colocalization with NK1R at the protein and mRNA transcript level, respectively (Fig. 2H,J). Quantification of Tacr1 and $t d T$ neurons in dual FISH experiments indicated that while Cre-mediated recombination is highly specific to Tacr 1 neurons $(88.5 \pm 6.3 \%$ of all $t d T$ neurons expressed Tacr 1$)$, it only occurs in roughly half of all Tacr1-expressing cells $(44.9 \pm 2.2 \%$ of all Tacrl cells expressed tdT; Fig. 2I) across dorsal horn spatial regions.

Dissecting the functional role of NK1R spinal neurons in itch required selective targeting of Tacr ${ }^{\mathrm{CreER}}$ neurons via local injection of Cre-dependent viruses into the spinal cord. As a first step, we asked whether virally-mediated Cre-dependent recombination in the Tacr ${ }^{\text {CreER }}$ mouse line recapitulated the spatial distribution of endogenous Tacrl expression. A Cre-dependent virus encoding a neuron-specific excitatory DREADD fused to an mCherry fluorescent reporter (Tacr1::hM3Dq-mCherry; AAV2hSyn-DIO-hM3Dq-mCherry) was injected into the L3/L4 spinal segment of Tacrl ${ }^{\text {CreER }}$ mice (Fig. $2 K$ ). We selected a Cre-dependent virus encoding a membrane bound receptor over a cytosolic reporter to allow for better visualization of hM3Dq-mCherry neuron processes. Lumbar spinal cord segments were collected from Tacr1::hM3Dq-mCherry mice and recombined spinal neurons were visualized using an anti-RFP antibody (Fig. 2L,M). The spatial distribution of hM3Dq-mCherry neurons closely resembled that of Tacr 1 cells, with $27.3 \pm 3.3 \%$ located in the $\mathrm{SDH}, 67.3 \pm 2.4 \%$ in the DDH, and $5.4 \pm 1.7 \%$ in the LSN (Fig. $2 N)$. Thus, Tacr1 ${ }^{\text {CreER }}$ captures a subpopulation of Tacrl spinal dorsal horn neurons that is representative of the spatial distribution of all Tacr1 neurons.

Notably, viral-vector mediated Cre-dependent recombination was largely restricted to the side ipsilateral to the intraspinal viral injection (Fig. 2O). Moreover, viral transduction in Tacr $1^{\text {CreER }}$ mice was restricted to the spinal cord, as hM3Dq-mCherry was not detected in the corresponding lumbar dorsal root ganglia (Fig. $2 P$ ), in agreement with previous evidence that the AAV2 viral serotype is not retrogradely transported along axons (Burger et al., 2004).

\section{Chemogenetic activation of Tacr1 ${ }^{\text {CreER }}$ spinal neurons preferentially increases itch}

Next, to complement our pharmacology studies, we tested whether chemogenetic activation of Tacr1 ${ }^{\text {CreER }}$ spinal neurons similarly increased itch-related behaviors. To selectively activate NK1R spinal neurons, Cre-dependent viruses encoding a neuron-specific excitatory DREADD fused to a fluorescent reporter (Tacr1::hM3Dq-mCherry; AAV2-hSyn-DIO-hM3Dq-mCherry) or a Cre-dependent control virus encoding only a fluorescent reporter (Tacr1::control; AAV2-hSyn-DIO- mCherry or AAV2EF1a-EYFP) were injected into the L3/L4 spinal segment of Tacr1 ${ }^{\text {CreER }}$ mice (Fig. 3A). In Tacr1::hM3Dq-mCherry mice, systemic administration of $\mathrm{CNO}$ significantly increased sitedirected biting in response to an intradermal injection of chloroquine into the calf compared with Tacrl::control mice (Fig. 3B, $C)$. The duration of cumulative chloroquine-induced calf biting in Tacr1::hM3Dq-mCherry mice was significantly correlated to the number of hM3Dq-mCherry neurons (Fig. 3D). Thus, a significant amount of the variability in the chloroquine-induced biting of Tacr1::hM3Dq-mCherry mice is explained by variability in viral transduction.

Given the long-standing evidence that NK1R neurons are required for pain behaviors following injury (Mantyh et al., 1997; Nichols et al., 1999; Vierck et al., 2003; Wiley et al., 2007), we next tested whether chemogenetic activation of Tacr $1^{\text {CreER }}$ spinal 
A Spatial distribution of

B

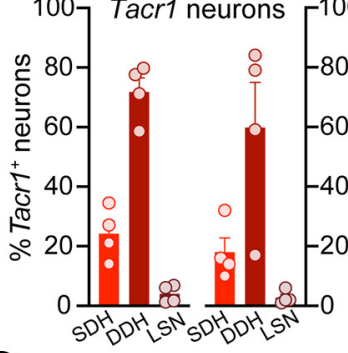

D Spatial distribution of

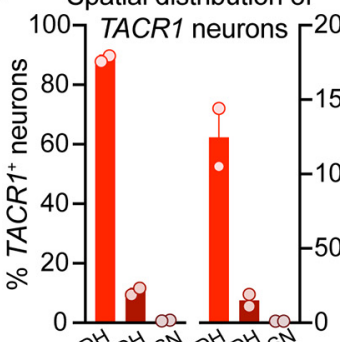

G

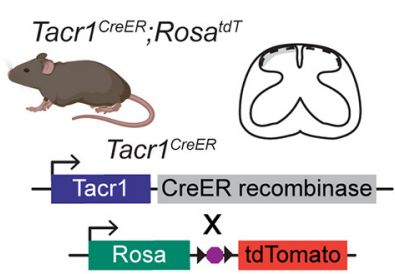

I Tacr1 ${ }^{\text {CreER }}$ efficiency

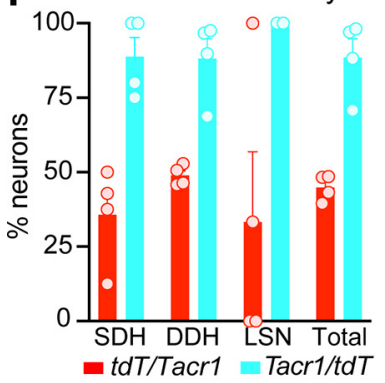

K

Tacr1::hM3Dq-mCherry

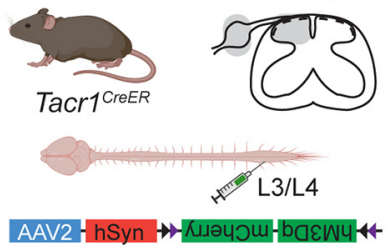

N

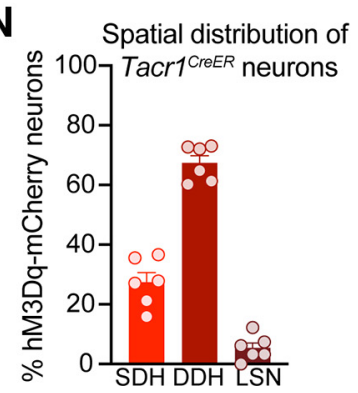

J

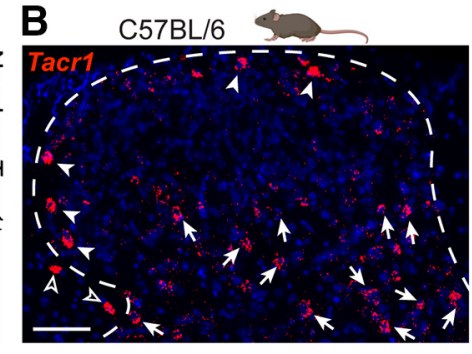

$E$

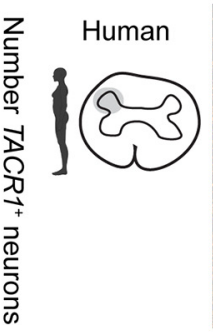

H
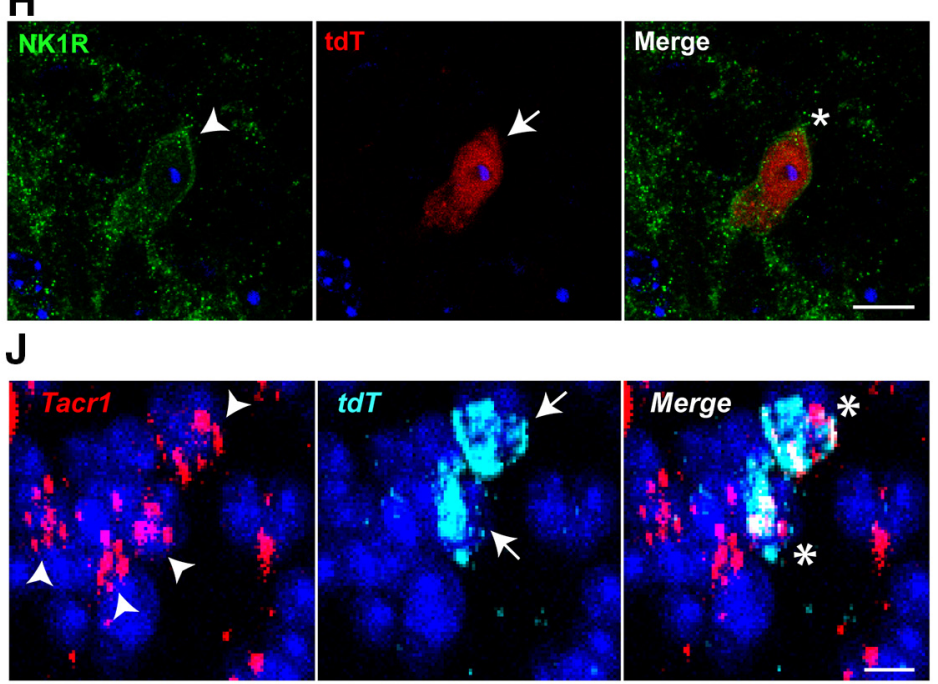

$\mathbf{L}$

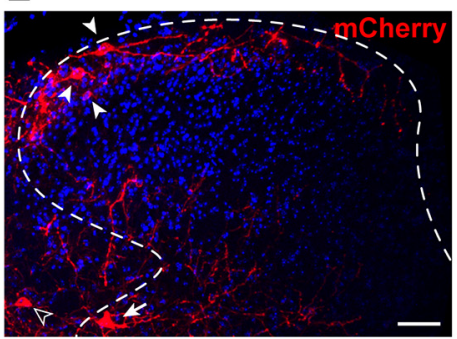

M

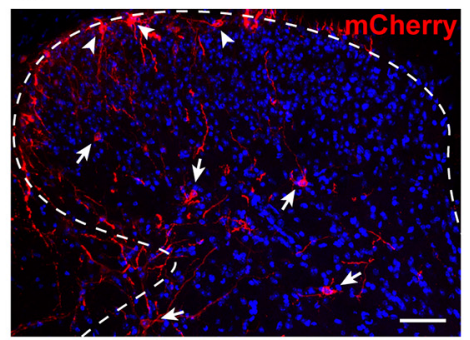

0

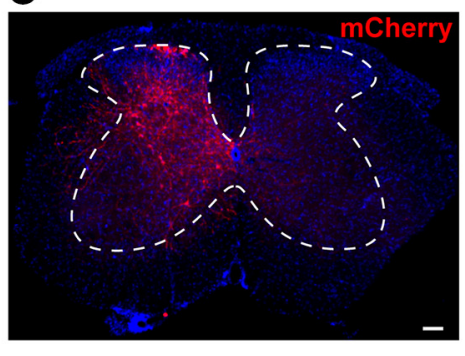

$\mathbf{P}$

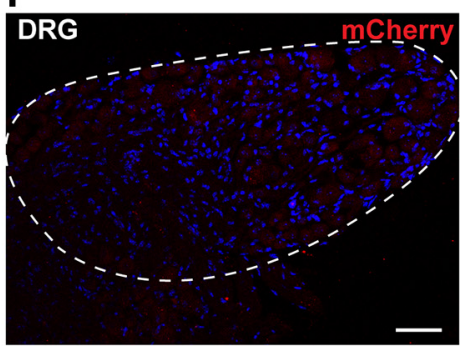

Figure 2. Characterization of Tacr1 expression in mouse and human spinal cord dorsal horn. A, Percentage (left $y$-axis) and corresponding total number (right $y$-axis) of Tacr1 neurons located within the SDH, DDH, and LSN of the mouse lumbar spinal cord ( $n=4$ mice, 2-3 hemisections/mouse), visualized via (B) FISH in C57BL/6 mice. Filled arrowheads, SDH; filled arrows, DDH; empty arrowheads, LSN. C, No Tacr1 signal was detected by FISH in the lumbar spinal cord of Tacr ${ }^{\text {Creek/(reek }}$ mice, which are equivalent to NK1R-/- mice. Scale bars: $50 \mu$ m (B, C). D, 
neurons also increased nociceptive or acute pain-related behaviors. CNO administration did not produce spontaneous hindlimb-directed pain or itch behaviors in Tacr1::hM3Dq-mCherry mice compared with Tacr1::control mice (Fig. 3E). Similarly, activation of Tacr $1^{\text {CreER }}$ spinal neurons did not alter nociceptive withdrawal thresholds to heat, cold, or mechanical stimuli (Fig. $3 F-H)$ compared with controls, as measured by the Hargreaves test, cold plantar assay, and von Frey test, respectively. These findings are consistent with previous reports that nociceptive withdrawal thresholds are unchanged by neurotoxic ablation of NK1R in the absence of injury (Mantyh et al., 1997; Nichols et al., 1999).

As a control for the possibility that the presence of the DREADD alone could influence baseline nociceptive withdrawal thresholds (Saloman et al., 2016), baseline thresholds of Tacr1:: control and Tacr1::hM3Dq mice were also measured in the absence of CNO. Expression of hM3Dq-mCherry alone did not affect baseline sensitivity to heat, cold, or mechanical stimuli compared with expression of control viruses (Fig. 3F-H). Moreover, $\mathrm{CNO}$ did not have a frank effect on nociception, as administration did not alter the withdrawal thresholds of the hindpaw contralateral to intraspinal viral injection in Tacr1:: Control or Tacr1::hM3Dq mice relative to baseline (Fig. 3M-O).

Lastly, using the intraplantar capsaicin model of neurogenic inflammation, we found that nocifensive behavioral responses such as licking or lifting of the hindpaw were unaffected by activation of Tacrl ${ }^{\mathrm{CrEER}}$ spinal neurons (Fig. 3I). These findings diverge from previous reports that NK1R-expressing neurons are required for capsaicin-induced nocifensive behaviors (Mantyh et al., 1997; Nichols et al., 1999; Vierck et al., 2003).

To confirm that the dose of CNO used in behavioral studies was sufficient to activate Tacr1::hM3Dq-mCherry neurons, we evaluated cFOS expression as a surrogate marker for neuronal activation across the spinal cord dorsal horn (Fig. 3J,K).

\section{$\leftarrow$}

Percentage (left $y$-axis) and corresponding total number (right $y$-axis) of TACR1 neurons located within the SDH, DDH, and LSN of the human cervical spinal cord ( $n=2$ donors, 3 hemisections/donor), visualized via $(\boldsymbol{E}, \boldsymbol{F})$ FISH. *Autofluorescence due predominantly to lipofuscin is shown to facilitate visualization of the substantia gelatinosa, bounded by the dotted lines. Scale bars: $250 \mu \mathrm{m}(\boldsymbol{E}), 50 \mu \mathrm{m}(\boldsymbol{F})$, and $25 \mu \mathrm{m}$ (Fi, Fii, insets). Filled arrowheads, SDH. G, To evaluate the efficiency and specificity of $\operatorname{Tacr}^{\text {(reER }}{ }^{\text {-mediated recombination within }}$ the lumbar spinal cord, $\operatorname{Tacr}^{{ }^{\mathrm{CreER}}}{ }_{;}$Ros $a^{\text {tdT }}$ mice were generated. $\boldsymbol{H}$, Representative IHC image showing colocalization of NK1R immunoreactivity (green) and $\mathrm{Tacr}^{\text {(reER }}$-mediated tdT expression (red) in an SDH neuron. Scale bar, $10 \mu \mathrm{m}$. Arrowhead or arrow, labeled neuron; Asterisk, dual-labeled neuron. I, Quantification of the colocalization of Tacr1 and tdT mRNA within SDH, DDH, and LSN neurons ( $n=4$ mice). J, Representative FISH image showing

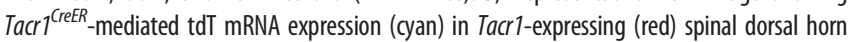
neurons. Scale bar, $10 \mu \mathrm{m}$. Arrowhead or arrow, labeled neuron; Asterisk, dual-labeled neuron. $\boldsymbol{K}, \operatorname{Tacr}^{\text {CreER }}$ mice received intraspinal injections of the Cre-dependent virus AAV2-hSynDI0-hM3Dq-mCherry to determine whether Cre-mediated recombination recapitulated the spatial distribution of endogenous Tacr1 expression. $\boldsymbol{L}, \boldsymbol{M}$, Representative IHC images of the spatial distribution of hM3Dq-mCherry neurons (red) throughout the spinal cord dorsal horn. Filled arrowheads, SDH; filled arrows, DDH; empty arrowheads, LSN. Scale bars: $50 \mu \mathrm{m}$. $\mathbf{N}$, Percentage of hM3Dq-mCherry neurons located within the SDH, DDH, and LSN of the lumbar spinal cord ( $n=6$ mice). 0 , Representative IHC image of the lumbar spinal cord showing that viral-vector-mediated Cre-dependent recombination in $\mathrm{TaCr}^{\mathrm{CreER}}$ mice, indicated by $m$ Cherry expression (red), was largely restricted to the side ipsilateral to the intraspinal viral injection. Scale bar, $50 \mu \mathrm{m}$. $\boldsymbol{P}$, Representative IHC image of the corresponding ipsilateral dorsal root ganglia (DRG), which lacks mCherry (red), indicating that viral vector-mediated Credependent recombination was restricted to the spinal cord of Tacr ${ }^{\text {CreER }}$ mice. Scale bar, $50 \mu \mathrm{m}$. FISH, fluorescence in situ hybridization; SDH, superficial dorsal horn; DDH, deeper dorsal horn; LSN, lateral spinal nucleus. Data in $\boldsymbol{A}, \boldsymbol{D}, \boldsymbol{I}, \boldsymbol{N}$ are shown as mean $\pm S E M$, with open circles representing individual mice.
CNO-induced cFOS expression was significantly higher in Tacr1::hM3Dq-mCherry neurons $(67.9 \pm 6.0 \%$ of total neurons) as compared with Tacr $1:$ :control neurons $(6.9 \pm 0.6 \%$ of total neurons; Fig. $3 L$ ). Collectively, these findings suggest that chemogenetic activation of $\mathrm{Tacr} 1^{\mathrm{CrEER}}$ spinal neurons preferentially increases itch behavior.

\section{Tacr1 ${ }^{\text {CreER }}$ captures predominantly interneurons}

Previous neurotoxic ablation studies were interpreted to support a role for NK1R spinal projection neurons in itch. However, data here suggest that ablation strategies would have likely targeted interneurons as well. To investigate this, we asked whether virally-captured neurons in Tacr1::hM3Dq-mCherry mice were spinal projection neurons or interneurons.

First, we leveraged AAV2-hSyn-DIO-hM3Dq-mCherry as an anterograde tracer and evaluated the brains of Tacr1::hM3DqmCherry mice for the central targets of ascending spinal projection neurons (Fig. 4A). In line with previous findings in rodents (Al-Khater and Todd, 2009; Cameron et al., 2015), the most robust hM3Dq-mCherry processes were observed in the contralateral $1 \mathrm{PBN}$, as well as the contralateral lateral and ventrolateral periaqueductal gray (PAG; Fig. 4B,C).

Next, we performed dual-labeling studies to distinguish between Tacr1::hM3Dq-mCherry spinal projection neurons and interneurons. Though anterograde tracing revealed hM3DqmCherry neuron processes in both the IPBN and the PAG, we chose to back label neurons from the IPBN because it has been shown to be the target of virtually all lumbar spinal projection neurons of the anterolateral tracts in rodents (Todd, 2010). To visualize spinoparabrachial neurons, the retrograde tracer CTB was stereotaxically injected into the IPBN on the side contralateral to the intraspinal viral injection (Fig. $4 D, H$ ), and the number of hM3Dq-mCherry and CTB-labeled neurons were quantified across the dorsal horn (Fig. 4E). Compared with previous reports estimating the total number of spinoparabrachial neurons within the SDH of the mouse lumbar spinal cord (9.9 CTB-labeled neurons/60 $\mu \mathrm{m}$; Cameron et al., 2015), we observed slightly fewer retrogradely labeled CTB neurons (5.9 CTB-labeled neurons/ $60 \mu \mathrm{m})$.

Throughout the dorsal horn, a small percentage of hM3DqmCherry neurons (SDH: 16.7\%, DDH: 1.9\%, LSN: 6.7\%) were CTB-backlabeled spinoparabrachial neurons, suggesting that Tacrl ${ }^{\text {CreER }}$ neurons are predominantly interneurons (Fig. $4 F, I, J$ ). This observation is striking as it calls into question whether NK1R is an acceptable marker for spinal projection neurons (Zhao et al., 2014; Cameron et al., 2015; Bardoni et al., 2019). While NK1R is expressed in spinal projection neurons, it is not exclusively expressed in spinal projection neurons.

Previous immunohistochemistry and in situ hybridization studies in wild-type mice estimate that between $65 \%$ and $90 \%$ of all mouse spinoparabrachial neurons express NK1R or Tacr1, respectively (Akiyama et al., 2015; Cameron et al., 2015; Häring et al., 2018). We report that a smaller percentage of CTB-backlabeled spinoparabrachial neurons (SDH: $32.1 \%$, DDH: $12.9 \%$, LSN: $16.7 \%$ ) coexpressed hM3Dq-mCherry (Fig. 4F). However, this observation is consistent with the efficiency of our genetic (Tacr $1^{\text {CreER }}$ allele) and viral tools for targeting NK1R spinal neurons.

Lastly, to determine whether CNO administration preferentially activated one Tacr1::hM3Dq-mCherry neuron population over another, we quantified the percentage of hM3Dq-mCherry and hM3Dq-mCherry, CTB neurons that were immunoreactive for cFOS (Fig. 4G). We found that cFOS expression was 

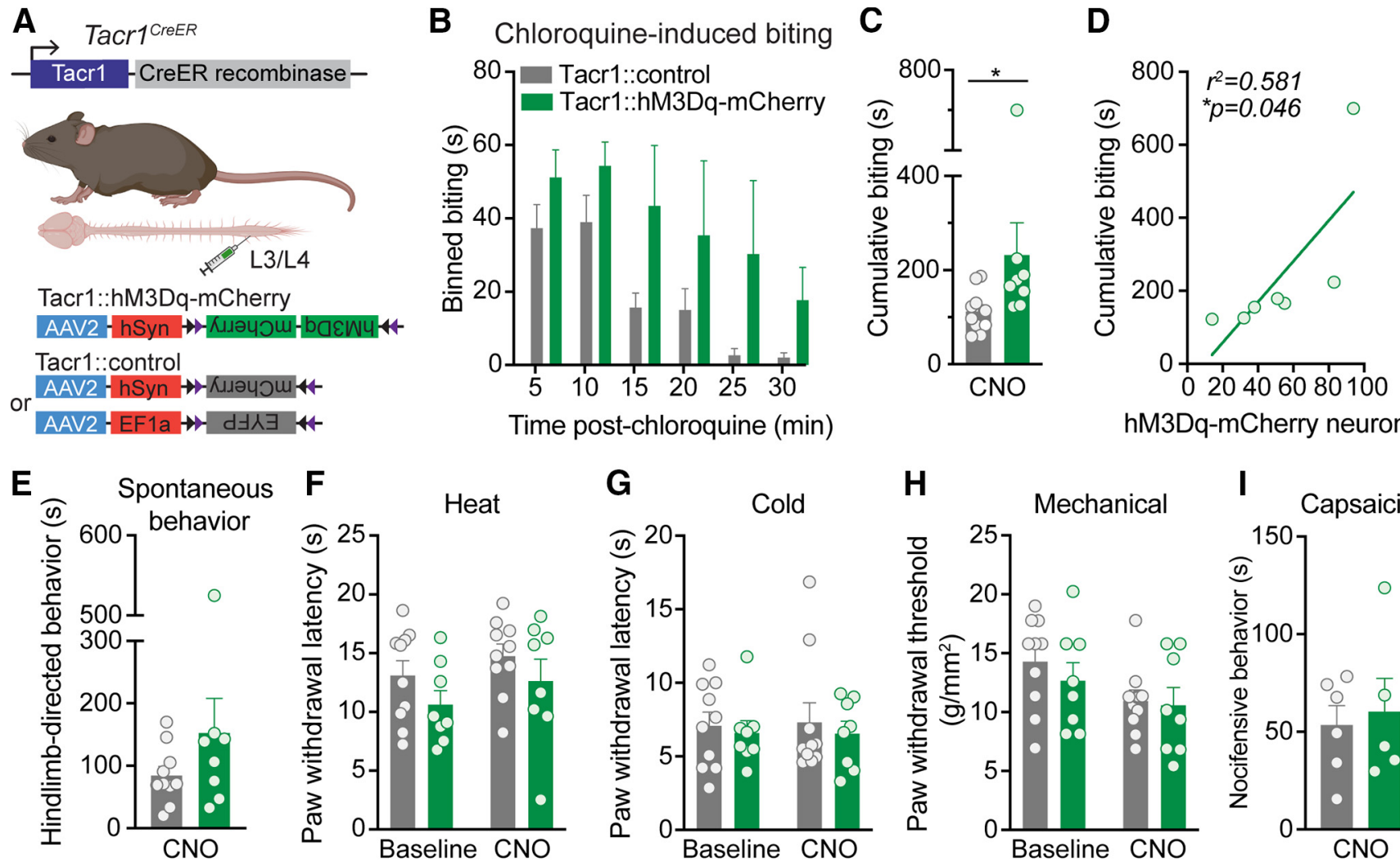

Time post-chloroquine ( $\mathrm{min})$

hM3Dq-mCherry neurons
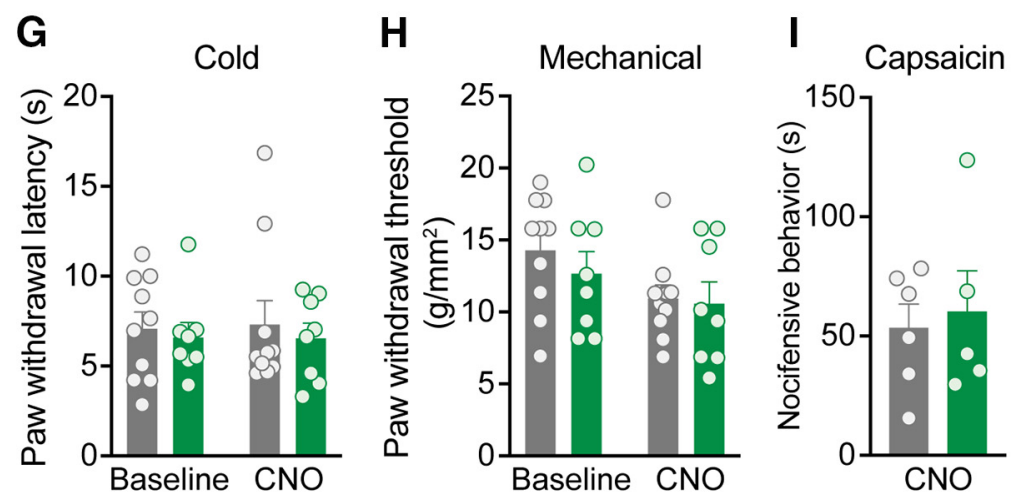

- Tacr1::control - Tacr1::hM3Dq-mCherry
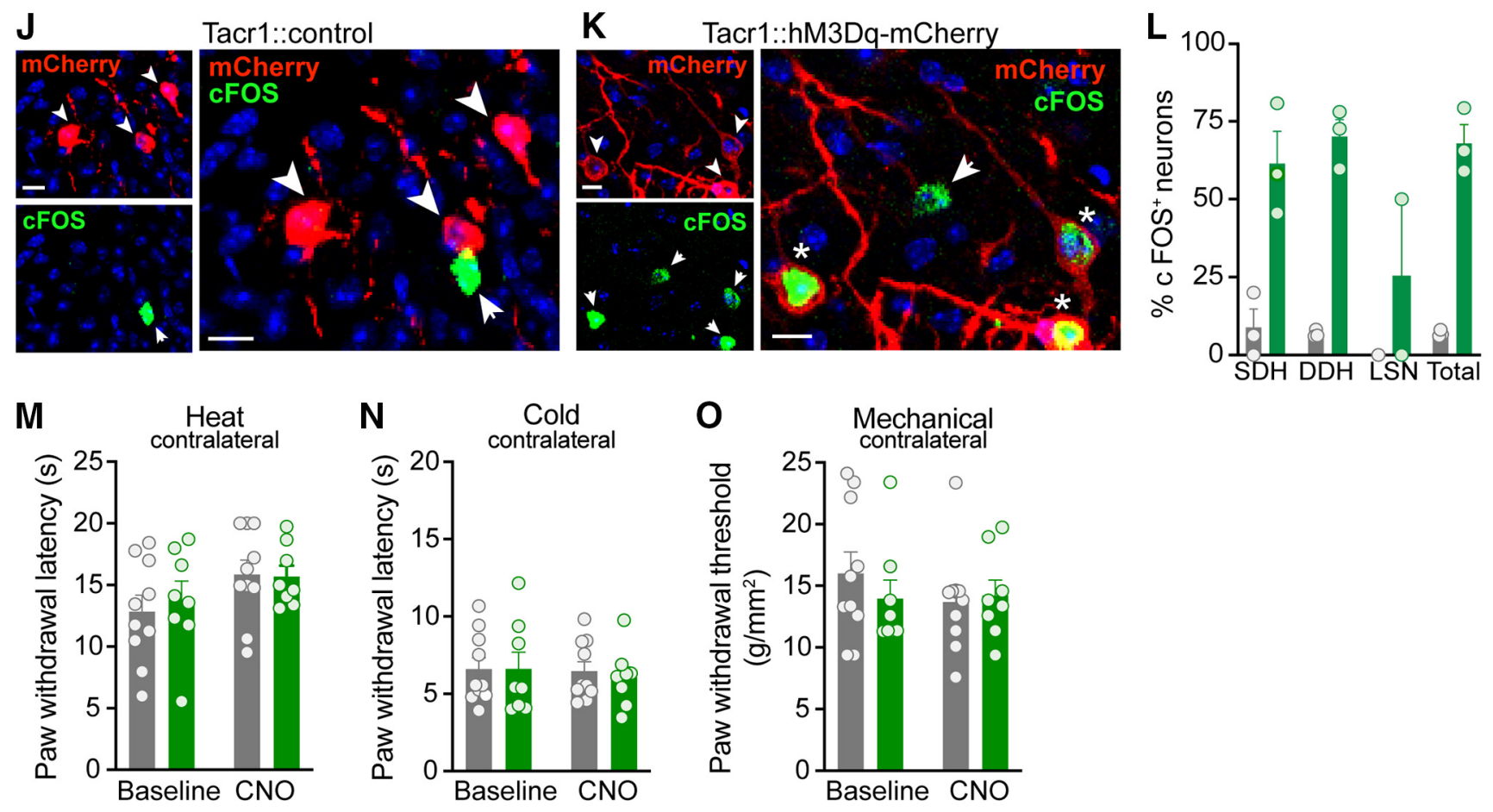

Figure 3. Chemogenetic activation of Tacr ${ }^{\text {CreeR }}$ spinal neurons preferentially increases itch. A, Strategy for selectively targeting Tacr ${ }^{\text {CreeR }}$ spinal neurons. To gain chemogenetic access to $\operatorname{Tacr}^{\text {CreeR }}$ spinal neurons, unilateral intraspinal viral injections of AAV2-hSyn-DI0-hM3Dq-mCherry (Tacr1::hM3Dq-mCherry) and either AAV2-hSyn-DI0-mCherry or AAV2-EF1a-DI0-EYFP (Tacr1:: control) were targeted to the lumbar dorsal horn of Tacr ${ }^{\text {(reeR }}$ mice. B, Duration of site-directed biting in response to an intradermal chloroquine injection (100 $\left.\mu \mathrm{g} / 5 \mu \mathrm{ll}\right)$ into the calf following systemic CNO administration (5 mg/kg, i.p.) in Tacr1::control and Tacr1::hM3Dq-mCherry mice, binned over time ( $n=8-10$ mice/group). C, CN0 administration significantly increased the cumulative duration of calf biting of Tacr1::hM3Dq-mCherry mice compared with Tacr1:::controls (Mann-Whitney $U$ test, ${ }^{*} p=0.0155, U=13, \mathrm{df}=16, n=8-10$ mice/group). D, The duration of cumulative chloroquine-induced biting in Tacr1::hM3Dq-mCherry mice was significantly correlated with the number of recombined hM3Dq-mCherry neurons (Pearson's correlation, ${ }^{*} p=0.0463$, $r^{2}=0.581, r=0.762, n=7$ mice). $\boldsymbol{E}$, Chemogenetic activation of Tacr ${ }^{\text {(reetR }}$ neurons with CNO did not affect pain or itch hindlimb-directed spontaneous behaviors of Tacr1::hM3Dq-mCherry mice compared with Tacr1::controls (Mann-Whitney $U$ test, $p=0.315, U=28, \mathrm{df}=16, n=8-10$ mice/group). Hindpaw nociceptive withdrawal thresholds to $(\boldsymbol{F})$ heat (two-way RM ANOVA, time $\times$ virus, $p=0.889, F_{(1,16)}=0.0202, n=8-10$ mice/group), $(\boldsymbol{G})$ cold (two-way RM ANOVA, time $\times$ virus, $p=0.886, F_{(1,16)}=0.0213, n=8-10$ mice/group), and $(\boldsymbol{H})$ mechanical (two- 
equivalent between hM3Dq-mCherry and hM3Dq-mCherry, CTB neurons, indicating that Tacrl::hM3Dq-mCherry interneurons and spinal projection neurons were equally activated by CNO.

\section{NK1R is expressed in GRPR interneurons within the SDH}

Based on the observations that NK1R spinal neurons modulate itch and that the majority of Tacr ${ }^{\text {CreER }}$ neurons manipulated in chemogenetic behavioral studies were interneurons, we wondered where NK1R interneurons fit within the current model of spinal itch circuitry. The current model of spinal itch transmission positions GRPR interneurons as a cellular gate of itch. Therefore, we asked whether NK1R is expressed in GRPR interneurons.

GRPR expression is restricted to neurons within the SDH (Sun and Chen, 2007; Sun et al., 2009; Bardoni et al., 2019), and thus coexpression analyses were similarly limited to the SDH in our studies. We first tested whether NK1R and GRPR mRNA transcripts are expressed within the same neurons. Dual FISH studies on lumbar spinal cord slices from C57BL/6 mice demonstrated notable overlap between Tacr1 and Grpr, with $48.1 \pm$ $4.3 \%$ of Tacr 1 SDH cells coexpressing Grpr, and $38.8 \pm 3.1 \%$ of Grpr SDH cells coexpressing Tacr1 (Fig. 5A,B).

To address whether NK1R and GRPR are functionally coexpressed within the same neurons, we decided to use two-photon $\mathrm{Ca}^{2+}$ imaging within an ex vivo spinal cord preparation, as NK1R and GRPR are both Gq-coupled GPCRs that cause release of intracellular $\mathrm{Ca}^{2+}$ stores on activation (Bordey et al., 1994; Karlsson and Ahrén, 1996). To determine the appropriate Cre driver for the genetically encoded $\mathrm{Ca}^{2+}$ indicator GCaMP6s, we performed dual FISH studies. These showed extensive colocalization of the glutamatergic neuronal marker Vglut2 with Tacr1 (99.0 $\pm 0.6 \%$ of Tacr1 neurons) and Grpr (98.5 $\pm 0.5 \%$ of Grpr neurons) in $\mathrm{SDH}$ neurons, indicating that these are excitatory neuron populations (Fig. $5 C-E$ ), in agreement with previous studies (Zhao et al., 2014; Häring et al., 2018; Freitag et al., 2019). Therefore, we selectively targeted Vglut2-positive excitatory neurons within the $\mathrm{SDH}$ in $\mathrm{Ca}^{2+}$ imaging experiments (Fig. $5 F$ ). To assess functional expression of NK1R and GRPR in SDH neurons, $\mathrm{Ca}^{2+}$ imaging experiments were performed in the presence

\footnotetext{
$\leftarrow$

way RM ANOVA, time $\times$ virus, $p=0.520, F_{(1,16)}=0.432, n=8-10$ mice/group) stimuli were unchanged following CNO administration in Tacr1::hM3Dq-mCherry mice compared with Tacr1:::controls. Across all nociceptive withdrawal tests, there was no main effect of virus (heat: $p=0.124, F=2.63$, cold: $p=0.592, F=0.299$, mechanical: $p=0.535, F=0.402$ ), indicating that hM3Dq-mCherry expression alone did not affect baseline nociceptive withdrawal thresholds compared with expression of control virus. I, CNO administration did not alter the duration of nocifensive behaviors in response to intraplantar capsaicin $(0.001 \%$ capsaicin, $10 \mu \mathrm{l}$ ) in Tacr1::hM3Dq-mCherry mice relative to Tacr1:::controls (Student's $t$ test, $p=0.729, t=0.358, \mathrm{df}=9, n=5-6$ mice/group). Representative IHC images of lumbar spinal cord sections demonstrating mCherry (red) and CFOS (green) expression in (J) Tacr1::control and (K) Tacr1::hM3Dq-mCherry mice 90 min following CNO administration, coinciding with the timeframe of behavioral testing. Scale bars, $10 \mu \mathrm{m}$. Arrowhead or arrow, labeled neuron; Asterisk, dual-labeled neuron. L, Quantification of CFOS + mCherry neurons shows that CNO induced significantly more CFOS expression in Tacr1::hM3Dq-mCherry neurons compared with Tacr1::neurons (mixed effects models, virus $\times$ spatial distribution, ${ }^{* *} p=0.0072$, $n=3$ mice/group). Chemogenetic activation of Tacr ${ }^{\text {(reeR }}$ neurons with CNO administration did not affect the contralateral hindpaw withdrawal thresholds of Tacr1::hM3Dq-mCherry mice to $(\boldsymbol{M})$ heat (two-way RM ANOVA, time $\times$ virus, $p=0.699, F_{(1,16)}=0.155, n=8-10$ mice/group), $(\boldsymbol{N})$ cold (two-way RM ANOVA, time $\times$ virus, $p=0.753, F_{(1,16)}=0.102, n=8-$ 10 mice/group), or (0) mechanical (two-way RM ANOVA, time $\times$ virus, $p=0.0 .338, F_{(1,16)}$ $=0.789, n=8-10$ mice/group) stimuli relative to Tacr1::controls. $\boldsymbol{B}, \mathbf{C}, \boldsymbol{E}-\mathbf{I}, \mathbf{L}-\mathbf{0}$, Data are shown as mean \pm SEM, with open circles representing individual mice.
}

of TTX so that neurons that directly responded to SP or GRP, and thus express NK1R or GRPR, respectively, could be identified (Fig. 5G,H).

In alignment with FISH analyses, we observed considerable overlap in responsivity to SP and GRP. Specifically, we found that out of all excitatory SDH neurons, $12.3 \%$ responded to both SP and GRP, while 9.4\% responded to SP alone, and 9.0\% responded to GRP alone (Fig. 5I). Analysis of the excitatory SDH neurons that responded to either agonist revealed that $56.9 \pm$ $10.5 \%$ of SP-responsive SDH neurons were also activated by GRP, and $58.8 \pm 9.3 \%$ of GRP-responsive SDH neurons were also activated by SP (Fig. $5 J$ ). Taken together, these results show that NK1R is functionally expressed within a subset of excitatory GRPR interneurons within the SDH. Importantly, this evidence demonstrates that NK1R is well positioned to modulate spinal itch transmission.

\section{Discussion}

The results of this study address two important questions regarding NK1R spinal dorsal horn neurons: (1) Is NK1R a selective marker for ascending spinal projection neurons? and (2) Where do NK1R neurons fit within the current model of spinal itch transmission?

\section{NK1R does not selectively mark spinal projection neurons}

Although previous reports have acknowledged that NK1R is expressed in spinal projection neurons, as well as interneurons (Todd et al., 1998; Al Ghamdi et al., 2009), NK1R is still frequently (and inappropriately) described as a selective marker for spinal projection neurons. Moreover, the presence or absence of NK1R immunoreactivity has been used as an inclusion criteria for determining whether a given dorsal horn neuron population includes spinal projection neurons (Zhao et al., 2014; Bardoni et al., 2019; Huang et al., 2019; Wercberger et al., 2020).

Here, we demonstrated that Tacr1 is expressed throughout the mouse dorsal horn: in SDH and DDH neurons, as well as the LSN. This spatial distribution was recapitulated in our Tacr ${ }^{\text {CreER }}$ mouse line, and retrograde labeling from the PBN in these mice shows that projection neurons represent a small fraction $(<10 \%)$ of all Tacr1 spinal neurons, highlighting that the majority of NK1R neurons are interneurons. In human, we similarly observed TACR1 expression throughout the dorsal horn, though in contrast to mouse, its expression appears to be highly restricted to the $\mathrm{SDH}$. While retrograde labeling studies are unfeasible in humans, given the sheer number of TACR1 neurons observed within the SDH, we speculate that TACR1 expression occurs in both spinal projection neurons and interneurons in human. Thus, while NK1R marks spinal projection neurons, it does not selectively mark spinal projection neurons.

The idea that NK1R selectively marks spinal projection neurons originates from a variety of circumstantial findings in rodents. For instance, neurotoxic ablation studies have shown loss of NK1R immunoreactivity that was largely limited to lamina I of the dorsal horn, where spinal projection neurons are most concentrated (Mantyh et al., 1997; Vierck et al., 2003). Furthermore, the finding that NK1R immunoreactivity is lower in interneurons than spinal projection neurons (Al Ghamdi et al., 2009) has been oversimplified, leading to the idea that NK1R immunoreactivity identifies projection neurons. Lastly, the use of NK1R as a selective projection neuron marker also stemmed from a lack of alternative markers. To date, NK1R remains the marker that captures the greatest proportion of projection 
A

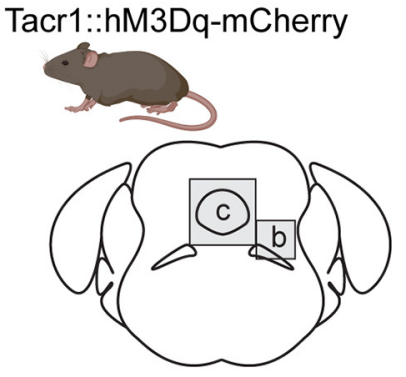

B

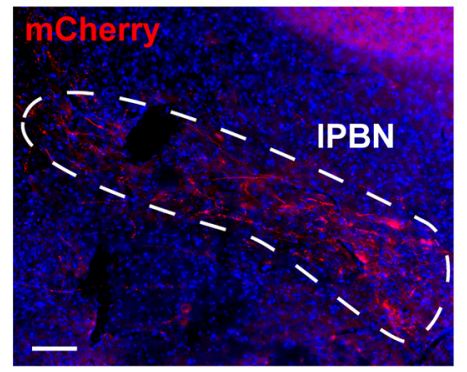

$\mathbf{F}$

E hM3Dq-mCherry, Ств

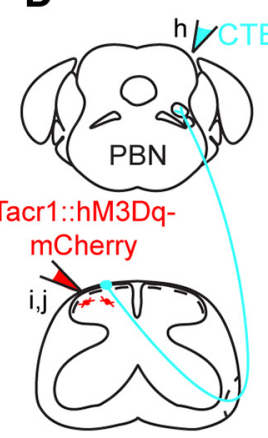

$\mathrm{H}$

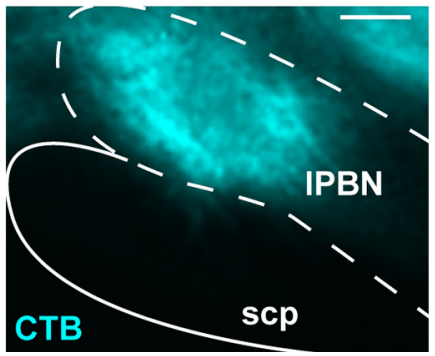

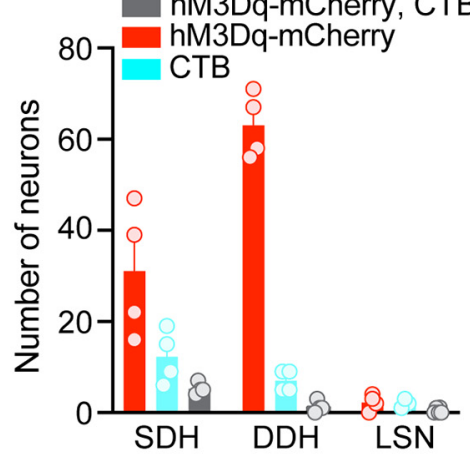

I

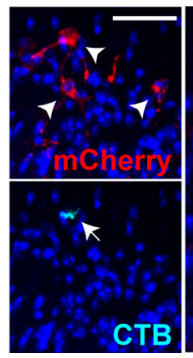

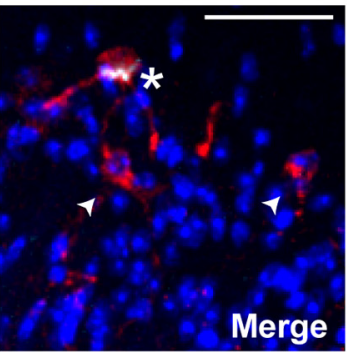
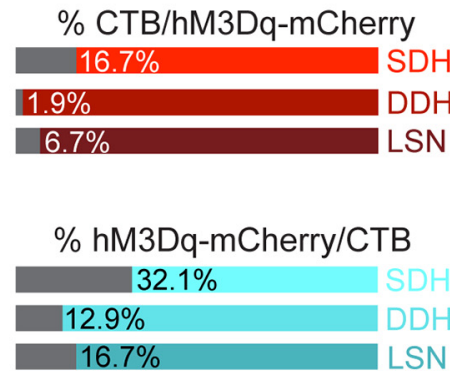

C
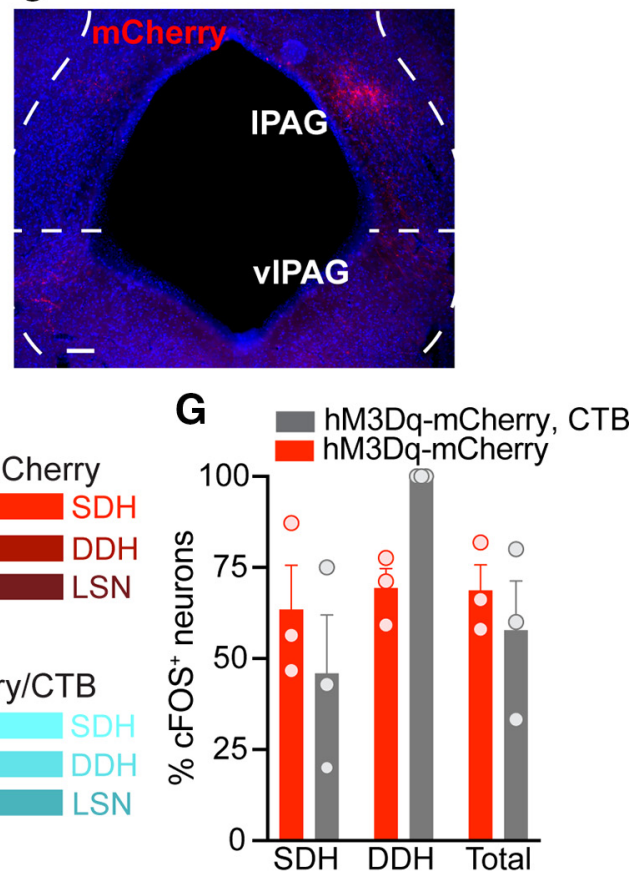

$J$

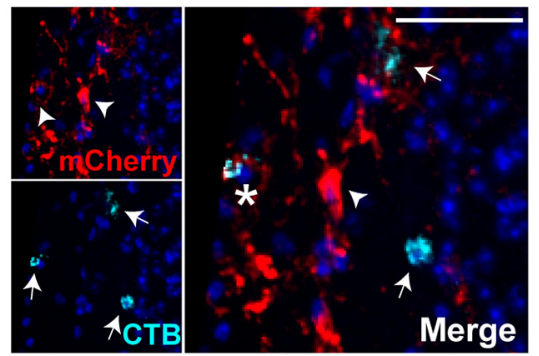

Figure 4. Tacr ${ }^{\text {CreeR }}$ spinal neurons are predominately interneurons. $A$, The brains of Tacr1::hM3Dq-mCherry neurons were collected and sectioned coronally to evaluate whether Tacr ${ }^{\text {CreeR }}$ captured spinal projection neurons. Representative immunostained coronal brain sections showing hM3Dq-mCherry processes (red) within the (B) IPBN, as well as the (C) IPAG and vIPAG contralateral to intraspinal viral injection. Scale bars: $50 \mu \mathrm{m}$. D. Experimental strategy to determine the relative proportion of Tacr1::hM3Dq-mCherry spinal projection neurons versus interneurons. The retrograde tracer CTB was injected into the contralateral IPBN of Tacr1::hM3Dq-mcherry mice to distinguish spinoparabrachial neurons (CTB and virally-mediated hM3Dq-mCherry expression) from interneurons (virally-mediated hM3Dq-mCherry expression only). $E$, Quantification of the total number of neurons labeled by hM3Dq-mCherry, CTB, or both within the SDH, DDH, and LSN of Tacr1::hM3Dq-mCherry mice, revealing that the majority of Tacr ${ }^{\text {(reeR }}$ spinal neurons are local interneurons $(n=4$ mice, 5 sections/mouse). F, Percentage of (top) total hM3DqmCherry neurons that were dual-labeled with CTB and (bottom) total CTB neurons that were dual-labeled with hM3Dq-mCherry within the SDH, DDH, and LSN, based off of number of neurons presented in $\boldsymbol{E}$. G, Quantification of the percentage of cFOS ${ }^{+}$hM3Dq-mCherry and hM3Dq-mCherry, CTB neurons across the dorsal horn shows equal activation following CNO administration (Student's $t$ test, $p=0.513, t=7.18, \mathrm{df}=4, n=3$ mice). Data are the same as those reported in Figure $3 K-L$ but were reanalyzed to evaluate cF0S immunoreactivity in interneurons versus spinoparabrachial neurons of Tacr1::hM3Dq-mCherry mice. cFOS expression was detected within a single LSN in one mouse (Fig. 3L), and thus LSN neurons are not included here. $\boldsymbol{H}$, Representative image of a targeted (TB injection (cyan) into the IPBN. Scale bar: $50 \mu \mathrm{m}$. I, J, Representative IHC images of lumbar spinal cord sections demonstrating the small extent of colocalization between hM3Dq-mcherry (red) and (TB (cyan). Scale bars, $10 \mu \mathrm{m}$. Arrowhead or arrow, labeled neuron; Asterisk, dual-labeled neuron. IPBN, lateral parabrachial nucleus; scp, superior cerebellar peduncle; IPAG, lateral PAG; vIPAG, ventrolateral PAG. SDH, superficial dorsal horn; DDH, deeper dorsal horn; LSN, lateral spinal nucleus. E, G, Data are shown as mean \pm SEM, with open circles representing individual mice.

neurons (an estimated 65-90\% in mouse), though recent single cell RNA-sequencing studies hold promise for novel spinal projection neuron markers, such as Lypd1 (Akiyama et al., 2015; Cameron et al., 2015; Häring et al., 2018). Overall, while these studies have been essential in identifying NK1R as a potential marker, the assertion that NK1R is a specific spinal projection marker is an oversimplification of this previous work.

\section{A potential role for NK1R interneurons in spinal itch transmission}

NK1R spinal neurons have previously been implicated in itch, but further characterization of these neurons and how they fit within the current model of spinal itch transmission has remained unexplored. Here, we provide evidence that pharmacological activation of spinal NK1R and chemogenetic activation of Tacrl ${ }^{\text {CreER }}$ spinal neurons potentiates acute itch behavior in mice, whereas pharmacological inhibition of spinal NK1R suppresses acute itch behavior, complementing previous NK1R neurotoxic ablation studies (Carstens et al., 2010; Akiyama et al., 2013, 2015; Acton et al., 2019).

A key finding of the present study is that NK1R interneurons likely contribute to spinal itch transmission. We showed that the majority of Tacrl ${ }^{\text {CreER }} \mathrm{SDH}$ neurons are interneurons, and that chemogenetic activation of these neurons increases behavioral responses to the pruritogen chloroquine. Further, our $\mathrm{Ca}^{2+}$ imaging findings indicate a subpopulation of excitatory neurons within the SDH functionally expresses NK1R, and at least half of 
A $\quad$ C57BL/6
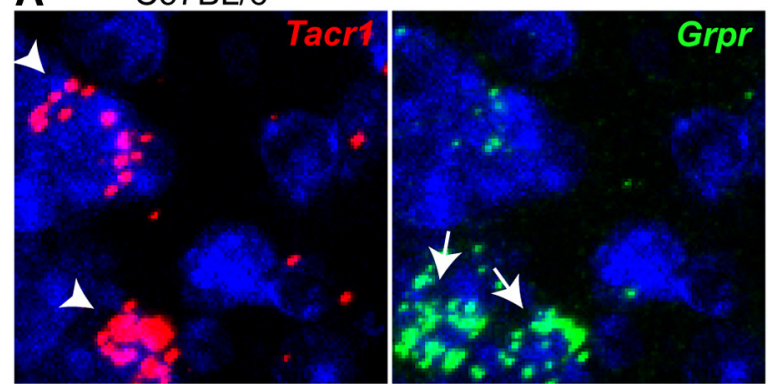

C

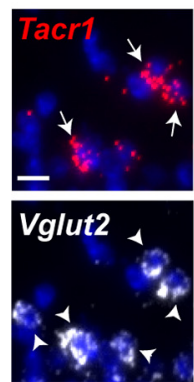

C57BL/6

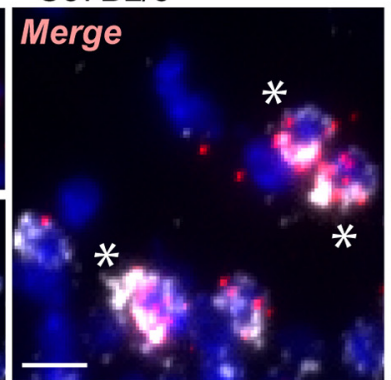

D

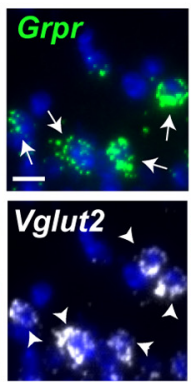

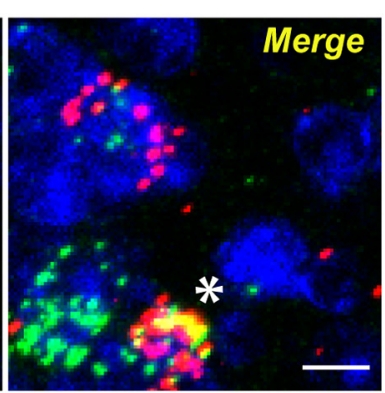

C57BL/6

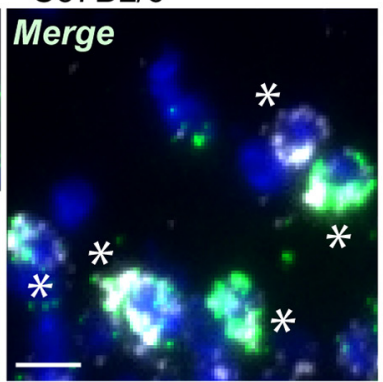

H

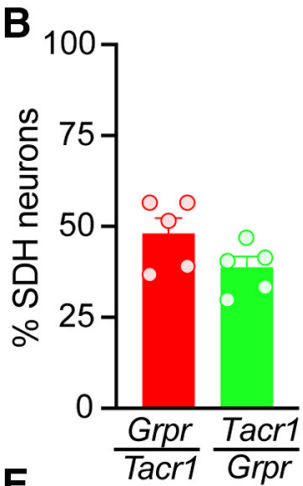

E

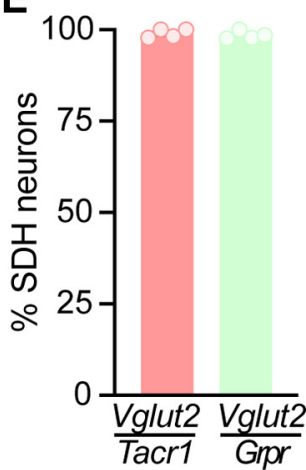

$\mathbf{F}$

G

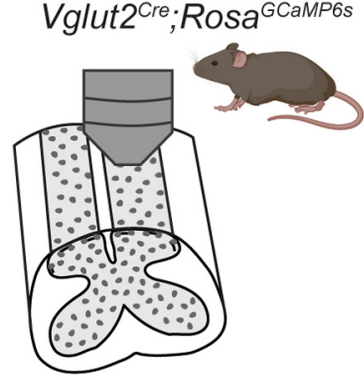

- Excitatory SDH neurons (GCaMP6s-labeled)

I

Excitatory SDH neurons

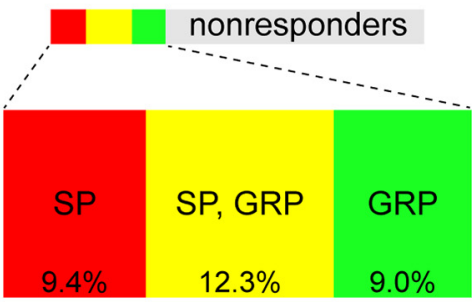

TTX

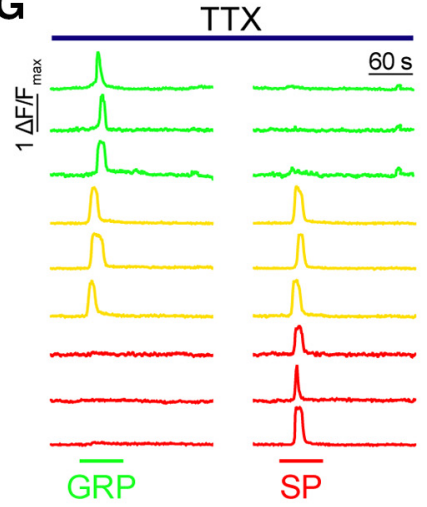

J

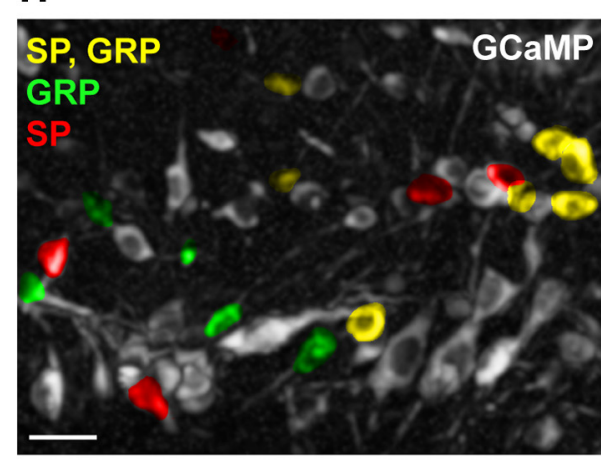

K

K Previous model Brain

Itch

Skin Spinal cord

lam I/Ilo

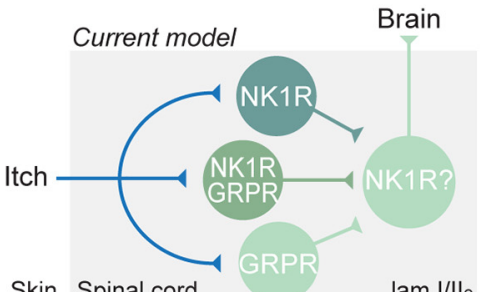

Figure 5. NK1R neurons in the SDH are a subset of GRPR interneurons. $A$, Representative images of dual FISH for Tacr1 (red) and Grpr (green) performed on lumbar spinal cord sections from C57BL/6 mice. Scale bar: $10 \mu \mathrm{m}$. B, Percentage of SDH neurons that coexpress Tacr1 and Grpr in C57BL/6 mice ( $n=5$ mice). Representative images of dual FISH evaluating the expression of the excitatory neuronal marker Vglut2 (white) in (C) Tacr1-expressing and (D) Grpr-expressing SDH neurons in C57BL/6 mice. Scale bars: $10 \mu \mathrm{m}$. E, Percentage of Tacr1 and Grpr SDH neurons that coexpress Vglut2 in C57BL/ 6 mice $\left(n=4\right.$ mice). $\boldsymbol{F}$, Schematic illustrating experimental set up for $\mathrm{Ca}^{2+}$ imaging of excitatory SDH neurons using Vglut2 ${ }^{\text {Gre }}$; Rosa ${ }^{\text {GCaMPos }}$ mice. $\mathbf{G}$, Representative traces of $\mathrm{Ca}^{2+}$ transients in response to $1 \mu \mathrm{m} \mathrm{SP}$ and $300 \mu \mathrm{m}$ GRP in the presence of $500 \mathrm{~nm}$ TX. $\boldsymbol{H}$, Representative psuedocolored fluorescent image showing Vglut ${ }^{\text {(ree }}$; Ros $a^{\text {GCamPGs }}$ SDH neurons (gray) activated by SP (red), GRP (green), or both (yellow). Scale bar: $25 \mu \mathrm{m}$. I, Treemap of the percentage of excitatory SDH neurons that responded to SP (red), GP (green), both SP and GRP (yellow), or neither (gray; $n=5$ mice, 57-189 Vglut2 $2^{\text {(re }}$;Rosa ${ }^{\text {GCaMPos }}$ neurons/mouse). J, Percentage of SDH neurons activated by both SP and GRP in Vglut2 $2^{\text {(ree }}$; Rosa ${ }^{\text {GCaMPos }}$ mice ( $n=5$ mice). $\boldsymbol{K}$, Schematic of the previous (top) and current (bottom) proposed model of the positions of NK1R and GRPR neurons within itch spinal circuitry. Solid lines do not necessarily represent direct synaptic connections. SDH, superficial dorsal horn; TIX, tetrodotoxin; SP, substance P; GRP, gastrin-releasing peptide. $\boldsymbol{A}, \boldsymbol{C}, \boldsymbol{D}$, Arrowhead or arrow, labeled neuron; Asterisk, dual-labeled neuron. $\boldsymbol{B}, \boldsymbol{E}, J$, Data are shown as mean \pm SEM, with open circles representing individual mice. 
these neurons coincide with GRPR interneurons, which are a critical hub for spinal itch transmission (Sun and Chen, 2007; Sun et al., 2009; Liu et al., 2019; Pagani et al., 2019).

One surprising observation from our study is different strategies for activating NK1R spinal neurons produced different effects on spontaneous itch behavior. While pharmacological activation of spinal NK1R elicited robust spontaneous itchrelated behaviors, chemogenetic activation of Tacr $1^{\text {CreER }}$ spinal neurons did not. One possible explanation for this observation is that NK1R and hM3Dq-although both Gq-coupled GPCRsengage different intracellular signaling pathways. Another striking finding was chemogenetic activation of Tacr $1^{\text {CreER }}$ spinal neurons increased itch, but not pain, behaviors. This finding might be because of the efficiency of the Tacr $1^{\text {CreER }}$ allele, which captures about half of all Tacr1 neurons, or possibly reflects that the allele preferentially targets a subset Tacr ${ }^{\mathrm{CreER}}$ spinal neurons that integrate itch.

It is well established that different forms of chemical itch (i.e., histaminergic, nonhistaminergic) are detected by distinct populations of primary afferent fibers (Dong and Dong, 2018), yet whether distinct spinal circuits contribute to the processing of histaminergic versus nonhistaminergic itch remains unclear. In our pharmacological inhibition and chemogenetic activation studies involving chloroquine, we provide evidence that NK1R neurons mediate nonhistaminergic itch. While histaminergic itch was not tested here, previous work has demonstrated NK1R antagonists delivered intrathecally preferentially block nonhistaminergic itch, suggesting SP-NK1R signaling may be restricted to nonhistaminergic itch (Akiyama et al., 2013).

NK1R is expressed in a subpopulation of GRPR interneurons Whether NK1R and GRPR are coexpressed within SDH neurons has been a controversial question. Previous histology studies on GRPR-eYFP mice reported NK1R and GRPR are nonoverlapping markers (Bardoni et al., 2019). In contrast, Tacr1 and Grpr were found to demarcate an excitatory neuron population ("Glut12") in recent unbiased classification of dorsal horn neuronal subtypes (Häring et al., 2018; Zeisel et al., 2018). Here, we provide evidence using FISH and $\mathrm{Ca}^{2+}$ imaging that the two receptors are coexpressed at the both the transcript and functional receptor level. Likewise, our behavioral findings point to functional overlap of NK1R and GRPR neurons in itch signaling, as the behavioral responses to intrathecal injection of SP, a selective NK1R agonist, and GRP closely resembled one another. One interpretation of this finding is that these behaviors reflect the activation of a common neural substrate for itch (e.g., NK1R/ GRPR interneurons) by the different agonists.

Importantly, the finding that NK1R is expressed within a subset of GRPR interneurons similarly positions NK1R in the center of spinal itch circuitry. Our results therefore suggest the previous model of spinal itch transmission, which proposed a linear relationship between GRPR interneurons and NK1R spinal projection neurons, requires updating. We propose that spinal integration of itch input by NK1R and GRPR neurons is far more complex, with several interneuron subpopulations-those expressing NK1R, GRPR, or both NK1R and GRPR_-integrating itch input, which is then likely relayed to the brain by NK1R spinal projection neurons (Fig. $5 K$ ).

Our data also indicate that within the SDH, NK1R and GRPR are not entirely overlapping populations; rather, approximately half of each population overlaps with the other. This finding suggests GRPR interneurons are a heterogenous population, as highlighted by recent electrophysiological characterization of GRPREFYP neurons (Bardoni et al., 2019; Pagani et al., 2019).
Whether NK1R/GRPR neurons convey itch through the same or different mechanisms as GRPR neurons lacking NK1R is an important question for future studies.

NK1R as a therapeutic target for itch-What have we learned? Our study provides evidence that NK1R spinal neurons mediate behavioral responses to itch in rodents. Taken together with additional studies showing NK1R blockade and ablation reduce acute and chronic itch in rodent models (Carstens et al., 2010; Akiyama et al., 2013, 2015; Acton et al., 2019), NK1R/GRPR interneurons are a plausible target for NK1R antagonists that were efficacious in reducing itch severity scores in Phase II clinical trials (Ständer et al., 2010, 2019a,b; Pereira and Ständer, 2018; Yosipovitch et al., 2018; Pojawa-Gołąb et al., 2019; Pariser et al., 2020). However, current NK1R antagonists appear to have limited clinical efficacy. The results of Phase III clinical trials are beginning to unfold, reporting NK1R antagonist treatment failed to significantly improve itch ratings relative to placebo (NCT03540160). Thus, it is becoming apparent that while NK1R certainly plays a part in itch, its role is likely a modulatory one.

It is interesting to compare the development of NK1R antagonists for the treatment of chronic pain and chronic itch. Analogous NK1R ablation and inhibition studies in rodents suggested NK1R as a therapeutic target for pain; however, NK1R antagonists failed broadly in clinical trials and NK1R was quickly abandoned as a therapeutic pain target (Hill, 2000; Boyce and Hill, 2004). NK1R antagonists have undoubtedly shown greater clinical efficacy in the treatment of chronic itch than chronic pain, yet are likely to be pulled once again from development. Although patients are itching for relief, the trials and tribulations of the development of NK1R as a therapeutic target for itch and pain may represent a cautionary tale that thorough preclinical testing is necessary before moving onto clinical trials.

Nonetheless, the findings of the present study highlight a potential new role for NK1R interneurons in itch, which comprise a subpopulation of excitatory GRPR interneurons. More broadly, this works adds to the current understanding of neurons that transmit itch within the spinal cord, which may in turn be leveraged for novel treatments in the future.

\section{References}

Acton D, Ren X, Di Costanzo S, Dalet A, Bourane S, Bertocchi I, Eva C, Goulding M (2019) Spinal neuropeptide Y1 receptor-expressing neurons form an essential excitatory pathway for mechanical itch. Cell Rep 28:625-639.e6.

Akiyama T, Tominaga M, Davoodi A, Nagamine M, Blansit K, Horwitz A, Carstens MI, Carstens E (2013) Roles for substance P and gastrin-releasing peptide as neurotransmitters released by primary afferent pruriceptors. J Neurophysiol 109:742-748.

Akiyama T, Nagamine M, Carstens MI, Carstens E (2014) Behavioral model of itch, alloknesis, pain and allodynia in the lower hindlimb and correlative responses of lumbar dorsal horn neurons in the mouse. Neuroscience 266:38-46.

Akiyama T, Nguyen T, Curtis E, Nishida K, Devireddy J, Delahanty J, Carstens MI, Carstens E (2015) A central role for dorsal horn neurons that express neurokinin-1 receptors in chronic itch. Pain 156:1240-1246.

Al Ghamdi KS, Polgár E, Todd AJ (2009) Soma size distinguishes projection neurons from neurokinin 1 receptor-expressing interneurons in lamina I of the rat lumbar spinal dorsal horn. Neuroscience 164:1794-1804.

Al-Khater KM, Todd AJ (2009) Collateral projections of neurons in laminae I, III, and IV of rat spinal cord to thalamus, periaqueductal gray matter, and lateral parabrachial area. J Comp Neurol 515:629-646.

Aresh B, Freitag FB, Perry S, Blümel E, Lau J, Franck MCM, Lagerström MC (2017) Spinal cord interneurons expressing the gastrin-releasing peptide receptor convey itch through VGLUT2-mediated signaling. Pain 158:945-961. 
Azimi E, Reddy VB, Shade K-TC, Anthony RM, Talbot S, Pereira PJS, Lerner EA (2016) Dual action of neurokinin-1 antagonists on Mas-related GPCRs. JCI Insight 1:e89362.

Azimi E, Reddy VB, Pereira PJS, Talbot S, Woolf CJ, Lerner EA (2017) Substance $\mathrm{P}$ activates Mas-related $\mathrm{G}$ protein-coupled receptors to induce itch. J Allergy Clin Immunol 140:447-453.e3.

Bardoni R, Shen KF, Li H, Jeffry J, Barry DM, Comitato A, Li YQ, Chen ZF (2019) Pain inhibits GRPR neurons via GABAergic signaling in the spinal cord. Sci Rep 9:15804.

Blizzard CA, Lee KM, Dickson TC (2016) Inducing chronic excitotoxicity in the mouse spinal cord to investigate lower motor neuron degeneration. Front Neurosci 10:1-8.

Bonin RP, De Koninck Y (2014) A spinal analog of memory reconsolidation enables reversal of hyperalgesia. Nat Neurosci 17:1043-1045.

Bonin RP, Bories C, De Koninck Y (2014) A simplified up-down method (SUDO) for measuring mechanical nociception in rodents using von Frey filaments. Mol Pain 10:26.

Bordey A, Feltz P, Trouslard J (1994) Mobilization of intracellular calcium by substance $p$ in a human astrocytoma cell line (U-373 MG). Glia 11:277283.

Boyce S, Hill R (2004) Substance P (NK1) receptor antagonists - analgesic or not? Tachykinins 164:441-453.

Brenner DS, Golden JP, Gereau RW (2012) A novel behavioral assay for measuring cold sensation in mice. PLoS One 7:e39765.

Brenner DS, Golden JP, Vogt SK, Gereau RW (2015) A simple and inexpensive method for determining cold sensitivity and adaptation in mice. J Vis Exp. Advance online publication. Retrieved March 17, 2015. doi: $10.3791 / 52640$.

Burger C, Gorbatyuk OS, Velardo MJ, Peden CS, Williams P, Zolotukhin S, Reier PJ, Mandel RJ, Muzyczka N (2004) Recombinant AAV viral vectors pseudotyped with viral capsids from serotypes 1,2 , and 5 display differential efficiency and cell tropism after delivery to different regions of the central nervous system. Mol Ther 10:302-317.

Cameron D, Polgár E, Gutierrez-Mecinas M, Gomez-Lima M, Watanabe M, Todd AJ (2015) The organisation of spinoparabrachial neurons in the mouse. Pain 156:2061-2071.

Carstens EE, Carstens MI, Simons CT, Jinks SL (2010) Dorsal horn neurons expressing NK-1 receptors mediate scratching in rats. Neuroreport 21:303-308.

Dong X, Dong X (2018) Peripheral and central mechanisms of itch. Neuron 98:482-494.

Freitag FB, Ahemaiti A, Jakobsson JET, Weman HM, Lagerström MC (2019) Spinal gastrin releasing peptide receptor expressing interneurons are controlled by local phasic and tonic inhibition. Sci Rep 9:16573.

Hachisuka J, Baumbauer KM, Omori Y, Snyder LM, Koerber HR, Ross SE (2016) Semi-intact ex vivo approach to investigate spinal somatosensory circuits. Elife 5:e22866.

Haenraets K, Albisetti GW, Foster E, Wildner H (2018) Adeno-associated virus mediated transgene expression in genetically defined neurons of the spinal cord. J Vis Exp. Advance online publication. Retrieved May 12, 2018. doi: $10.3791 / 57382$.

Hargreaves K, Dubner R, Brown F, Flores C, Joris J (1988) A new and sensitive method for measuring thermal nociception. Pain 32:77-88.

Häring M, Zeisel A, Hochgerner H, Rinwa P, Jakobsson JET, Lönnerberg P, La Manno G, Sharma N, Borgius L, Kiehn O, Lagerström MC, Linnarsson S, Ernfors P (2018) Neuronal atlas of the dorsal horn defines its architecture and links sensory input to transcriptional cell types. Nat Neurosci 21:869-880.

Hill R (2000) NK1 (substance P) receptor antagonists - Why are they not analgesic in humans? Trends Pharmacol Sci 21:244-246.

Huang H, Kuzirian MS, Cai X, Snyder LM, Cohen J, Kaplan DH, Ross SE (2016) Generation of a NK1R-CreER knockin mouse strain to study cells involved in Neurokinin 1 receptor signaling. Genesis 54:593-601.

Huang T, Lin S, Malewicz NM, Zhang Y, Zhang Y, Goulding M, Lamotte RH, Ma Q (2019) Identifying the pathways required for coping behaviours associated with sustained pain. Nature 565:86-90.

Hylden JLK, Wilcox GL (1981) Intrathecal substance P elicits a caudallydirected biting and scratching behavior in mice. Brain Res 217:212-215.

Jendryka M, Palchaudhuri M, Ursu D, van der Veen B, Liss B, Kätzel D, Nissen W, Pekcec A (2019) Pharmacokinetic and pharmacodynamic actions of clozapine-N-oxide, clozapine, and compound 21 in DREADDbased chemogenetics in mice. Sci Rep 9:4522.
Kalueff AV, Tuohimaa P (2004) Grooming analysis algorithm for neurobehavioural stress research. Brain Res Protoc 13:151-158.

Kalueff AV, Stewart AM, Song C, Berridge KC, Ann M, Fentress JC (2016) Neurobiology of rodent self-grooming and its value for translationsl neuroscience. Nat Rev Neurosci 17:45-59.

Kardon AP, Polgár E, Hachisuka J, Snyder LM, Cameron D, Savage S, Cai X, Karnup S, Fan CR, Hemenway GM, Bernard CS, Schwartz ES, Nagase H, Schwarzer C, Watanabe M, Furuta T, Kaneko T, Koerber HR, Todd AJ, Ross SE (2014) Dynorphin acts as a neuromodulator to inhibit itch in the dorsal horn of the spinal cord. Neuron 82:573-586.

Karlsson S, Ahrén B (1996) Gastrin-releasing peptide mobilizes calcium from intracellular stores in HIT-T15 cells. Peptides 17:909-916.

Karlsson S, Ahrén B (1999) Cytosolic Ca2+ oscillations by gastrin releasing peptide in single HIT-T15 cells. Peptides 20:579-587.

LaMotte RH, Shimada SG, Sikand P (2011) Mouse models of acute, chemical itch and pain in humans. Exp Dermatol 20:778-782.

Lay M, Dong X (2020) Neural mechanisms of itch. Annu Rev Neurosci 43:187-205.

Liu MZ, Chen XJ, Liang TY, Li Q, Wang M, Zhang XY, Li YZ, Sun Q, Sun YG (2019) Synaptic control of spinal GRPR+ neurons by local and longrange inhibitory inputs. Proc Natl Acad Sci USA 116:27011-27017.

Mantyh PW, Demaster E, Malhotra A, Ghilardi JR, Rogers SD, Mantyh CR, Liu H, Basbaum AI, Vigna SR, John E, Mantyh PW, Demaster E, Malhotra A, Ghilardi JR, Rogers SD, Mantyh CR, Simone DA (1995) Receptor endocytosis and dendrite reshaping in spinal neurons after somatosensory stimulation. Science 268:1629-1632.

Mantyh PW, Mantyh PW, Rogers SD, Honore P, Allen BJ, Ghilardi JR, Li J, Daughters RS, Lappi DA, Wiley RG, Simone DA (1997) Inhibition of hyperalgesia by ablation of lamina I spinal neurons expressing the substance $P$ receptor. Science 278:275-280.

Marvizón JC, Martínez V, Grady EF, Bunnett NW, Mayer EA (1997) Neurokinin 1 receptor internalization in spinal cord slices induced by dorsal root stimulation is mediated by NMDA receptors. J Neurosci 17:8129-8136.

Nakatsuka T, Chen M, Takeda D, King C, King J, Xing H, Ataka T, Vierck C, Yezierski R, Gu JG (2005) Substance P-driven feed-forward inhibitory activity in the mammalian spinal cord. Mol Pain 1:20.

Nichols ML, Allen B, Rogers S, Ghilardi JR, Honore P, Luger NM, Finke MP, Li J, Lappi DA, Simone DA, Mantyh PW (1999) Transmission of chronic nociception by spinal neurons expressing the substance $\mathrm{P}$ receptor. Science 286:1558-1562.

Njoo C, Heinl C, Kuner R (2014) In vivo SiRNA transfection and gene knockdown in spinal cord via rapid noninvasive lumbar intrathecal injections in mice. J Vis Exp. Advance online publication. Retrieved Mar 22, 2014. doi: 10.3791/51229.

Pagani M, Albisetti GW, Sivakumar N, Wildner H, Santello M, Johannssen HC, Zeilhofer HU (2019) How gastrin-releasing peptide opens the spinal gate for itch. Neuron 103:102-117.e5.

Pariser DM, Bagel J, Lebwohl M, Yosipovitch G, Chien E, Spellman MC (2020) Serlopitant for psoriatic pruritus: a phase 2 randomized, doubleblind, placebo-controlled clinical trial. J Am Acad Dermatol 82:13141320.

Peirs C, Williams SPG, Zhao X, Walsh CE, Gedeon JY, Cagle NE, Goldring AC, Hioki H, Liu Z, Marell PS, Seal RP (2015) Dorsal horn circuits for persistent mechanical pain. Neuron 87:797-812.

Pereira MP, Ständer S (2018) Novel drugs for the treatment of chronic pruritus. Expert Opin Investig Drugs 27:981-988.

Pojawa-Gołąb M, Jaworecka K, Reich A (2019) NK-1 receptor antagonists and pruritus: review of current literature. Dermatol Ther (Heidelb) 9:391-405.

Polgár E, Wright LL, Todd AJ (2010) A quantitative study of brainstem projections from lamina I neurons in the cervical and lumbar enlargement of the rat. Brain Res 1308:58-67.

Ruzza C, Rizzi A, Malfacini D, Cerlesi MC, Ferrari F, Marzola E, Ambrosio C, Gro C, Severo S, Costa T, Calo G, Guerrini R (2014) Pharmacological characterization of tachykinin tetrabranched derivatives. Br J Pharmacol 171:4125-4137.

Saloman JL, Scheff NN, Snyder LM, Ross SE, Davis BM, Gold MS (2016) GiDREADD expression in peripheral nerves produces ligand-dependent analgesia, as well as ligand-independent functional changes in sensory neurons. J Neurosci 36:10769-10781. 
Samineni VK, Grajales-Reyes JG, Sundaram SS, Yoo JJ, Gereau RW (2019) Cell type-specific modulation of sensory and affective components of itch in the periaqueductal gray. Nat Commun 10:4356.

Sanders KM, Sakai K, Henry TD, Hashimoto T, Akiyama T (2019) A subpopulation of amygdala neurons mediates the affective component of itch. J Neurosci 39:3345-3356.

Seybold VS, Hylden JLK, Wilcox GL (1982) Intrathecal substance P and somatostatin in rats: behaviors indicative of sensation. Peptides 3:49-54.

Spike RC, Puskár Z, Andrew D, Todd AJ (2003) A quantitative and morphological study of projection neurons in lamina I of the rat lumbar spinal cord. Eur J Neurosci 18:2433-2448.

Ständer S, Luger TA (2015) NK-1 antagonists and itch. Handb Exp Pharmacol 226:237-255.

Ständer S, Siepmann D, Herrgott I, Sunderkötter C, Luger TA (2010) Targeting the neurokinin receptor 1 with aprepitant: a novel antipruritic strategy. PLoS One 5:e10968.

Ständer S, Kwon P, Hirman J, Perlman AJ, Weisshaar E, Metz M, Luger TA (2019a) Serlopitant reduced pruritus in patients with prurigo nodularis in a phase 2, randomized, placebo-controlled trial. J Am Acad Dermatol 80:1395-1402.

Ständer S, Spellman MC, Kwon P, Yosipovitch G (2019b) The NK1 receptor antagonist serlopitant for treatment of chronic pruritus. Expert Opin Investig Drugs 28:659-666.

Sun YG, Chen ZF (2007) A gastrin-releasing peptide receptor mediates the itch sensation in the spinal cord. Nature 448:700-703.

Sun YG, Zhao ZQ, Meng XL, Yin J, Liu XY, Chen ZF (2009) Cellular basis of itch sensation. Science 325:1531-1534.

Todd AJ (2010) Neuronal circuitry for pain processing in the dorsal horn. Nat Rev Neurosci 11:823-836.

Todd A, Spike R, Polgár E (1998) A quantitative study of neurons which express neurokinin-1 or somatostatin sst2a receptor in rat spinal dorsal horn. Neuroscience 85:459-473.
Todd AJ, McGill MM, Shehab SAS (2000) Neurokinin 1 receptor expression by neurons in laminae I, III and IV of the rat spinal dorsal horn that project to the brainstem. Eur J Neurosci 12:689-700.

Vierck CJ, Kline IV, RH, Wiley RG (2003) Intrathecal substance P-saporin attenuates operant escape from nociceptive thermal stimuli. Neuroscience 119:223-232.

Wang X, Marvizón JCG (2002) Time-course of the internalization and recycling of neurokinin 1 receptors in rat dorsal horn neurons. Brain Res 944:239-247.

Wercberger R, Braz JM, Weinrich JA, Basbaum AI (2020) Pain and itch processing by subpopulations of molecularly diverse spinal and trigeminal projection neurons. bioRxiv. doi: https://doi.org/10.1101/2020.06.17 156091

Wiley RG, Kline IR, Vierck CJ (2007) Anti-nociceptive effects of selectively destroying substance $\mathrm{P}$ receptor-expressing dorsal horn neurons using [Sar9,Met(O2)11]-substance P-saporin: behavioral and anatomical analyses. Neuroscience 146:1333-1345.

Yosipovitch G, Ständer S, Kerby MB, Larrick JW, Perlman AJ, Schnipper EF, Zhang X, Tang JY, Luger T, Steinhoff M (2018) Serlopitant for the treatment of chronic pruritus: results of a randomized, multicenter, placebo-controlled phase 2 clinical trial. J Am Acad Dermatol 78:882-891.e10.

Zeisel A, Hochgerner H, Lönnerberg P, Johnsson A, Memic F, van der Zwan J, Häring M, Braun E, Borm LE, La Manno G, Codeluppi S, Furlan A, Lee K, Skene N, Harris KD, Hjerling-Leffler J, Arenas E, Ernfors P, Marklund U, Linnarsson S (2018) Molecular architecture of the mouse nervous system. Cell 174:999-1014.e22.

Zhao ZQ, Wan L, Liu XY, Huo FQ, Li H, Barry DM, Krieger S, Kim S, Liu ZC, Xu J, Rogers BE, Li YQ, Chen ZF (2014) Cross-inhibition of NMBR and GRPR signaling maintains normal histaminergic itch transmission. J Neurosci 34:12402-12414. 